(From the R. Spreckels Physiological Laboratory of the University of California, Berkeley, California.)

\title{
Versuche über die Giftigkeit des Seewassers für Süsswassertiere (Gammarus pulex De Geer). \\ Von
}

\section{Wolfgang Ostwald.}

(Mit 2 Textfiguren und Tafel II-VII.)

Die vorliegende Untersuchung war in der Absicht begonnen worden, einige Einblicke in die Gesetzmässigkeiten und näheren Ursachen der Anpassung von Tieren an verschiedene Konzentrationen des umgebenden füssigen Mediums, zunächst von Süsswassertieren an Seewasser, zu erlangen. Dabei ergab sich aber die Notwendigkeit, vorher die Vorgänge der Giftwirkungen plötzlicher Konzentrationsänderungen sowie deren Ursachen einem näheren Studium zu unterziehen. Die bisherige Literatur über dieses letztere Problem beschränkt sich entweder auf die Feststellung der Lebenszeit von Süsswassertieren, die in Meerwasser gebracht wurden ${ }^{1}$ ) (Paul Bert, Plateau usw.), meist nur bei einer Konzentration und ohne Angabe des Salzgehaltes, oder aber bezieht sich auf Lösungen von Salzen, welche nur $z u m$ Teil im Seewasser vorkommen (Yasuda, Massart usw.). Untersuchungen über den Einfluss der einzelnen im Seewasser enthaltenden Salze, speziell in ihrer relativen Konzentration, welche also ein speziell bi ologis ches Interesse hätten, fehlen, ebenso exaktere quantitative

1) Die hierhergehörige Literatur ist so oft zusammengestellt worden, dass eine Wiederholung Platzverschwendung usw, bedeuten würde. Von solchen Zusammenstellungen an leicht zugänglichen Orten seien genannt: Semper, Existenzbedingungen der Tiere Teil I S. 174 ff. u. Anmerk. z. V. Kap. Leipzig 1880. Davonport, Experimental Morphology Part I p. 1-96, Part II p. 293-349. 1897-1899. - v. Fürth, Vergleichende chemische Physiologie usw. S. $622 \mathrm{ff}$. Jena 1903. 
Feststellungen über die Abhängigkeit der Giftwirkung des Seewassers von der Konzentration desselben.

Sodann bedurften die näheren Ursachen oder die Dynamik dieser Giftwirkungen einer weiteren Analyse. Die meisten älteren Autoren und selbst noch v. Fürth in seinem ausgezeichneten Buche sind der Ansicht, dass die giftige Eigenschaft des Salzwassers zum grössten Teile, wenn nicht ausschliesslich in seiner Hypertonizität besteht, d. h. dass der Entzug von Wasser seitens der hypertonisehen Lösung den Tod des Tieres bedinge. Obgleich diese Ansicht von der mehr "physikalischen" Natur dieser Giftwirkungen ja sehr wahrscheinlich ist, lassen sich doch mehrere Bedenken gegen sie geltendmachen. So ist z. B. durch J. Loeb ${ }^{\mathbf{1}}$ ) gezeigt worden, dass bei den entgegengesetzten Giftwirkungen, beim plötzlichen Hereinbringen von Seewasseramphipoden (Allorchestis sp.) in Süsswasser resp. destilliertes Wasser, keineswegs, wie bisher allgemein geglaubt wurde, die Wasseraufnahme seitens des Tieres der einzige schädliche Faktor ist, sondern dass mit grosser Wahrscheinlichkeit einige zur Erhaltung des Lebens nötige Salze aus dem Organismus herausgehen. Diese Ansicht wird unterstützt insbesondere auch durch die Untersuchungen $u$. a. von Fredericq ${ }^{2}$ ) und Quinton ${ }^{3}$ ), von denen besonders der letztere die Durchgängigkeit der äusseren Membranen von Seetieren nicht nur für Wasser, sondern auch für Salze, wie dies für pflanzliche, künstliche Membranen sowie solche höherer Tiere ja schon früher bekannt war, zeigte. Der Gedanke lag nahe, auch im umgekehrten Falle, bei den Wirkungen des Seewassers auf Süsswassertiere, ähnliche Verhältnisse zu finden. Es ergab sich nun bei Anwendung genauerer quantitativer Methoden in der Tat, dass die Vorstellung von der "rein physikalischen ${ }^{4}$ ) Natur der Salzwirkung nicht den Tatsachen entsprechend ist, dass sich vielmehr die toxischen Wirkungen des Seewassers (in ähnlicher Weise wie bei den Parallelversuchen Loeb's die Giftigkeit des Süsswassers) aus mehreren einzelnen Faktoren zusammensetzen. Durch speziellere quantitative Untersuchungen wurde eine zunächst rein empirische Analyse und Deutung dieser Wirkungen versucht.

1) J. Loeb, Pflüger's Archiv Bd. 97 S. $394 \mathrm{ff}, 1903$.

2) Fredericq, Bull. de l'Ácad, roy. de Belgique 1901 p. $68 \mathrm{ff}$.

3) Quinton, Compt. rend. t. 131 p. 952 ff. 1900.

4) v: Fürth, Chem. Physiologie S. 629 . Jena 1903. 


\section{I. Über die Abhängigkeit der Giftigkeit des Seewassers von seiner Konzentration.}

1. Versuchstechnik. Als Material diente Gammarus pulex De Geer. Um erstens eine möglichst grosse Gleichmässigkeit des Materials zu erzielen sowie zweitens um auf eventuelle Unterschiede der Geschlechter aufmerksam zu werden, wurden zu den Versuchen nur kopulierende Paare, die sich in ziemlich grosser Zahl fanden, benutzt. Es ist anzunehmen, dass diese Tiere gleichmässiger in physischer Beziehung beschaffen sind als beliebig ausgewählte, sowie, dass sie normale und sich in gutem Gesundheitszustande befindliche Organismen darstellen.

Die Konzentration wurde in der Weise verändert, dass zu dem Süsswasser, in dem die Tiere monatelang in Gefangenschaft lebten, in verschiedenen Versuchen verschieden viel Seewasser aus dem Hafen von San Francisco (bei Oakland) zugesetzt wurde. Der Salzgehalt dieses Wassers wurde nach sorgfältiger Bestimmung des spezifischen Gewichtes mittelst sehr genauer Aräometer nach der Formel

$$
\left.S=1310\left(S p \cdot \frac{17,5^{0}}{17,5^{0}}-1\right)^{1}\right)
$$

berechnet. Das spezifische Gewicht betrug bei $17,5^{\circ} 1,018$, der Salzgehalt also 23,6\%. Da es sich im Laufe der Untersuchung zeigte, dass eine Vermehrung der Konzentration des Wassers bis auf diesen Salzgehalt nicht genügte, um genauere Resultate über die Abhängigkeit der Giftigkeit von der Konzentration zu erhalten, mussten noch konzentriertere Lösungen angewendet werden. Diese erhielt ich, indem ich Oakland-Seewasser in grossen, flachen Schalen bis zur Hälfte seines Volumens verdunsten liess und nun mit diesem doppeltkonzentrierten Seewasser die Versuche in gleicher Anordnung fortsetzte ${ }^{2}$ ). Ich zog diese Methode dem Einkochen des Seewassers

1) Siehe z. B. Supan, Physische Erdkunde, 2. Aufl, S. 214. 1896.

2) Die Konzentrationserhöhung geschah in folgender Weise :

$$
\begin{aligned}
& \text { I. } 90 \text { Teile Süsswasser }+10 \text { Teile Seewasser }
\end{aligned}
$$

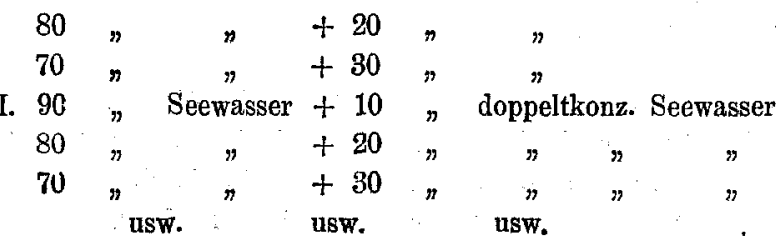


vor, um nicht in gleich starkem Masse die Bikarbonate des Seewassers in Karbonate zu verwandeln. Fs stellte sich indessen heraus, dass auch auf diesem Herstellungswege das doppeltkonzentrierte Seewasser ausgesprochen alkalisch reagierte. Eine nähere Untersuchung des gewöhnlichen Seewassers in den grossen Aquarien des Instituts ergab jedoch, - dass auch dieses, namentlich am Tage, schwach alkalisch reagierte. Loeb und $\mathrm{C}_{0} \mathrm{ttre} \mathrm{ll}^{1}$ ) haben für normales Oakland-Seewasser festgestellt, dass es nicht oder kaum alkalisch reagiert; der erstere fand indessen; dass eine deutliche Alkalinität zutage trat, wenn sich Algen (Ulva usw.) in den Gefässen befanden ${ }^{2}$ ). Dies letztere war entsprechend auch in dem Seewasser, dass zu meinen Versuchen verwendet wurde, der Fall gewesen.

Nach dem Ausprobieren mehrerer Versuchsanordnungen wurde als die beste und ökonomischste folgende angewendet: Die Pärchen wurden mittelst der Pipette auf ein trocknes Handtuch gebracht, welches das Wasser ziemlich schnell aufsaugte und so die Tiere mit ziemlicher Vollkommenheit trocknete. Ich fand, dass eine derartige kurze Behandlung die Lebensdauer der Tiere nicht feststellbar beeinflusste; ich habe im Gegenteil Tiere noch nach vielleicht zwei bis drei Stunden auf dem Handtuch und in der Luft befindlich lebend gefunden. Da unter anderem die Untersuchungen von Bullot ${ }^{3}$ ) über die Giftigkeit des destillierten Wassers ergeben hatten, dass sowohl die Menge der Flüssigkeit als auch die Anzahl der Tiere, die gleichzeitig in ein Gefäss getan werden, einen Finfluss auf ihre Lebensfähigkeit haben, wưden bei den endgültigen Versuchen überall zehn Pärchen in $100 \mathrm{ccm}$ Lösung getan. Leider musste ich bei den letzten Versuchen wegen Mangels an Material diese Zahl auf acht und sechs verringern; ich glaube indessen, dass speziell bei den verwendeten $h o ̈ h$ eren Konzentrationen und bei den entsprechend ku r zen Lebenszeiten der hier in Frage kommende Einfluss ein nicht sehr grosser gewesen sein wird. Als Zeitpunkt des Todes wurde das Aufhören der Kiemenbewegungen und der Bewegungsfähigkeit der Schwimmbeine und Antennen auch bei Berührung und starkem Drücken angenommen. Es wurde nämlich beobachtet, dass die Beweglichkeit der Kiemenblättchen in den niederen Konzentrationen

1) Pflüger's Archiv Bd. 99 S. 637. 1903.

2) Pflü ger's Archiv Bd. 101 S. 347. 1904.

3) Bullot, University of Calif. Public. Physiology vol. 1 p. $199 \mathrm{ff} .1904$. 
länger vorhanden war als die Beweglichkeit der Beine und Antennen, im Gegensatz zu dem Verhalten in konzentrierteren Lösungen, in welchen oft fünf bis zehn Minuten nach Erlöschen der Kiemenbewegungen spontane Bewegungen der Beine sich zeigten ${ }^{1}$ ). Die Tiere wurden gleichzeitig hereingebracht, die Lebenszeit jedes einzelnen gemessen und von allen sodann der Durchschnitt genommen. Bei sämtlichen Versuchen wurde, um die Variabilität der Resultate nicht noch weiter zu vermehren, kein Futter gegeben. Vorversuche zeigten, dass die Tiere circa drei Wochen obne dasselbe in reinem Süsswasser leben konnten.

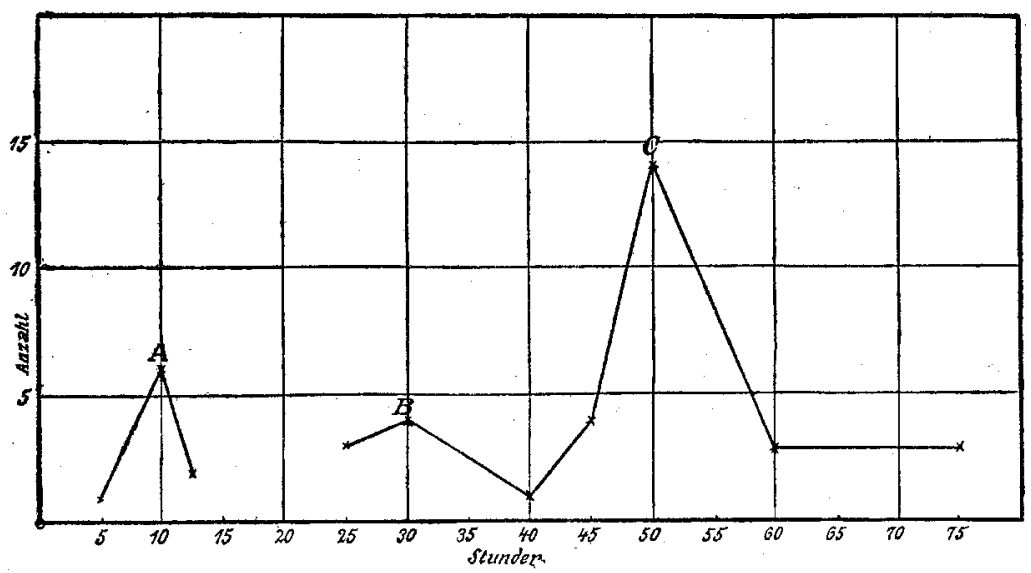

Fig. 1.

Da die Versuche quantitativer Art sein sollten, so waren die vorhandenen Variabilitätsfaktoren möglichst sorgfältig in Rechnung zu ziehen. Der erste und vielleicht wichtigste Faktor ist die Variabilität der Tiere selbst. Durch die Auswahl von Pärchen wurde versucht, diesen Faktor möglichst einzuschränken. Was die Variabilität der Männchen und Weibchen an und für sich anbetrifft, so soll beistehende Figur 1 z. B. die Art der Variabilität von Männchen in einem Salzwasser von ca. $2 \%$ veranschaulichen. Die Figur wurde erhalten, indem die gewonnenen Zahlen für die Lebensfähigkeit in variationsstatistischer Weise nach der Häufigkeit ihres Vorkommens eingetragen wurden. Es ergaben sich drei Gipfel $A, B$

1) Die Bewegung der Kiemenblättchen kann oft gleich nach ihrem Stillstand durch Drücken mit einer Pipette uuf den Vorderleib des Tieres künstlich auf ein paar Sekunden hervorgerufen werden. 
und $C$, von denen $A$ relativ am isoliertesten ist, wie dies in der Figur durch Unterbrechen der Linie ausgedrückt wurde. Ich glaube, die Deutung dieser drei Gipfel besteht darin, dass $A$ die kranken, vielleicht bei der Überführung und dem Trocknen verletzten Individuen darstellt, $B$ diejenigen, welche sich gerade während der Häutung befinden, und $C$ als der grösste Gipfel die Summe der tatsächlich normalen Tiere. Zu bemerken ist, dass nur bei den relativ verdünnten Lösungen und entsprechend längerer Lebenszeit der Tiere eine derartige mehrgipflige Variabilität sich feststellen liess. Bei höherer Konzentration ergab sich, abgesehen von ein paar beschädigten, sehr bald sterbenden Individuen, in der Regel nur ein Gipfel.

Ein zweiter Variabilitätsfaktor ist die Unsicherheit in der genauen Bestimmung des Todeseintrittes; diesen Faktor glaube ich durch die sehr grosse Häufigkeit der Beobachtungen und die dadurch erworbene Übung eingeschränkt zu haben. Drittens ist der Einfluss der Temperatur zu berücksichtigen. Obgleich ich nicht in einem Thermostaten gearbeitet habe, bin ich sicher, dass die Temperatur während dieses ersten Teiles meiner Untersuchungen in Aubetracht erstens des bekannten gleichmässigen Klimas dieses Teiles von Kalifornien sowie zweitens des Umstandes, dass die Versuche fast ausschliesslich zu derselben Tageszeit ausgeführt wurden, nicht um $5^{0}$ variiert hat. Da überdies dieser Einfluss der Temperatur speziell untersucht, werden soll, habe ich ihn hier vernachlässigt. Gelegentliche, zu anderen Zwecken unternommene Versuche zu verschiedenen Tageszeiten (mittags, nachts usw.) ergaben sowieso eine nur unbedeutende Variation des Lebenszeiten in dem hier in Betracht kommenden Temperaturgebiet.

Im allgemeinen glaubte ich indessen dem quantitativen Charakter dieser Untersuchungen am besten gerecht zu werden dadurch, dass möglichst zahlreiche und oft $\mathrm{zu}$ verschiedenen Zeiten und mit verschiedenen Fängen von Material wiederholte Versuche angestellt wurden. So wurden hier für die Bestimmung der Kurve der Abhängigkeit der Giftigkeit des Seewassers von seiner Konzentration über 900 Lebenszeiten zu verschiedenen Zeiten usw. gemessen.

2. Resultate. Die Resulte der Messungen der Abhängigkeit der Giftigkeit des Seewassers von seiner Konzentration werden durch die beifolgenden Kurven dargestellt. Die Abszisse stellt die Könzentrationen, die Ordinate die Lebenszeit dar. 
Ein auffälliges Verhalten ergab sich gleich bei den ersten Versuchen: der Umstand, dass sich Männchen und Weibchen in bezug auf ihre Lebenszähigkeit sehr verschieden verhalten. .Ohne Ausnahme erwiesen sich die Weibchen empfindlicher gegen den Salzeinfluss als die Männchen. Es sei gleich hier bemerkt, dass dies Verhalten sich auch bei den später zu beschreibenden Versuchen mit verschiedenen Salzgemischen durchgehend als gültig erwies; oft war die Empfindlichkeit eine grössere und annähernd parállele zu der des Männchens, zuweilen aber besass sie ihre spezifische Form.

Die Kurven auf Tafel II beginnen mit einer Konzentration von $25,9 \%$ 10 Teilen doppeltkonzentriertem Oaklandseewasser. Fig. 2 veranschaulicht, warum eine so hohe Konzentration zum Ausgangspunkt gewählt wurde.

Es zeigt sich nämlich, dass in den Salzkonzentrationen bis zu der des Seewassers die Giftigkeit noch eine so geringfügige ist, dass eine genanere Messung, wie sie hier angestrebt wurde, nicht möglich ist. Denn es ist zu erwarten und oben gezeigt worden, dass die Variabilität in der Lebensfähigkeit sowie sämtlicher einzelner physischer Eigenschaften mit der Zeit zunimmt, während welcher sie dem Versuch oder der Beobachtung unterworfen sind. Die Anzahl der Möglichkeiten, welche eine Variation bedingen können, wächst im entgegengesetzten Sinne wie die Zeit. In den Konzentrationen bis $50 \mathrm{ccm}$ Süsswasser und $50 \mathrm{cem}$ Seewasser zeigte sich überhaupt kein Einfluss; die Tiere leben ebenso lange wie in reinem Süsswasser. Erst von 20 Teilen Süsswasser und 80 Teilen Seewasser ab begann ein deutlicher schädlicher Einfluss. Indessen waren die erhaltenen Zahlen einerseits noch so gross, variierten anderseits so stark, dass ich diese Konzentrationen einstweilen aus meinen Versuchen ausschloss. Eine quantitative Bestimmung der Giftigkeit dieser Konzentrationen ist nur auf Grund eines ausserordentlich grossen Versuchsmaterials (eventuell mit Hilfe von graphischer Darstellung) oder aber bei höherer Temperatur möglich.

Während also für die Konzentrationen bis ungefähr zum reinen Seewasser mit einem Salzgehalt von $23,6^{\circ}{ }_{000}$ die Giftigkeit zuerst gleich Null und dann nur sehr geringfügig ist, nimmt sie bei den Konzentrationen 23,6 und $25,96 \%$ in ganz rapider Weise für beide Geschlechter zu, dabei für das Weibchen etwas früher als für das Männchen. Die Lebensdauer fällt beim Männchen von ca. 5-6 Tagen hei einer Konzentrationserhöhung von 23,6 auf 25,96 herab auf 
46 Stunden, beim Weibchen von ca. 29 auf $5^{1 / 2}$ Stunden, das ist beim letzteren ungefähr auf den fünften bis sechsten Teil. Von 25,6-28,3\% beträgt beim Weibchen der Unterschied nur $11 / 2$ Stunde, beim Männehen noch $40^{1 / 2}$ Stunden, d. h. der achte Teil. Von $28,3 \%$ ab findet indessen die Abnahme allmählicher und geringfügiger statt, bis beide Kurven von etwa $40,1 \%$ ab geradlinig und asymptotisch zur Abszisse verlaufen.

Der Unterschied zwischen Männchen und Weibchen nimmt ziemlich stetig ab mit steigender Konzentration. Während er bei normalem Seewasser ein paar Tage, bei einer Konzentration von 25,941 Stunden beträgt, verschwindet er beinahe bei doppeltkonzentriertem Seewasser $(47,2 \%$ ) und vollständig etwa bei $56,8 \%$.

Die genauen Resultate sind in folgender Tabelle enthalten:

Tabelle 1. Seewasser.

\begin{tabular}{|c|c|c|c|}
\hline $\begin{array}{c}\text { Konzentration } \\
\text { in } \% 000\end{array}$ & $\begin{array}{l}\text { Lebenszeit des } \\
\text { Weibchens } \\
\text { in Minuten }\end{array}$ & $\begin{array}{l}\text { Lebenszeit des } \\
\text { Männchens } \\
\text { in Minuten }\end{array}$ & Mittlere Lebenszeit \\
\hline $\begin{array}{l}58,7 \\
47,2 \\
44,8 \\
42,5 \\
40,1 \\
37,7 \\
35,4 \\
33,0 \\
30,6 \\
28,3 \\
25,9 \\
29,6\end{array}$ & $\begin{array}{c}36 \\
46 \\
50 \\
50 \\
53 \\
67 \\
77 \\
86 \\
127 \\
185 \\
328 \\
\text { ca. 29 Stunden }\end{array}$ & $\begin{array}{c}36 \\
48 \\
60 \\
75 \\
79 \\
101 \\
120 \\
147 \\
192 \\
326 \\
\text { ca. 46 Stunden } \\
\text { 5-6 Tage }\end{array}$ & $\begin{array}{c}36 \\
47 \\
55 \\
60,5 \\
66 \\
84 \\
98,5 \\
116,5 \\
159,5 \\
255,5 \\
\text { ca. } 20^{3 / 4} \text { Stunden } \\
3-4 \text { Tage. }\end{array}$ \\
\hline
\end{tabular}

Eine für die ganze Kurve gültige Formel habe ich bis jetzt noch nicht berechnen können.

Interessant ist es, dass die Versuche von J. Loeb ${ }^{1}$ ) über die Giftigkeit des Süsswassers auf Seewasser-Amphipoden insofern ein analoges Verhalten aufweisen, als auch hier "up to a certain limit of dilution the duration of life decreases but little with additional dilution; - - then, however, from a certain limit on, the decrease in the duration of life takes place very rapidly." Es scheint mir wahrscheinlich, dass dieser in beiden Fällen gleichartige steile Abfall der Kurven auch eine gemeinsame Ursache, die in beiden Fällen in einem Koagulationsvorgang,

1) J. Loeb, University of California Public. Physiol. vol. 1 p. 58.

E. P fl ü ger, Archiv für Physiologie. Bd. 106. 
welch letzterer bekanntlich $\mathrm{ja}$ in analoger Weise bei Verdünnung oder Konzentrationserhöhung der im Sol vorhandenen Elektrolyte plötzlich auftreten kann, bestehen wird, besitzt. Doch sollen derartige theoretische Erwägungen erst später und insbesondere auf Grund weiterer Experimente versucht werden.

\section{II. Über die Dynamik der Giftwirkungen des Seewassers anf Sïsswasser-Gammarus.}

Als rationellster, wenn auch vielleicht langweiligster Weg, in die nähere Dynamik dieser Giftwirkungen etwas einzudringen, erschien mir eine systematische und ausführliche Untersuchung der toxischen Wirkungen der einzelnen chemischen Komponenten des Seewassers sowie der mit diesen möglichen Variationen und Kombinationen. Es bestätigte sich dabei, wie ich glaube, die Erwartung, dass insbesondere grössere zusammenhängende Reihen gleichartiger Messungen, welche entsprechend graphisch dargestellt werden konnten, für den vorliegenden Zweck die geeignetsten Untersuchungsformen sind.

Die hauptsächlichsten, hier zunächst in Betracht kommenden Salze sind entsprechend den van't Hoff'schen Untersuchungen in folgendem Molekularverhältnis im Meerwasser enthalten :
I. $\mathrm{NaCl}=100$, $\mathrm{MgCl}_{2}=7,8$, $\mathrm{KCl}=2,1$, $\mathrm{MgSO}_{4}=\cdot 3,8$,$$
\mathrm{CaCl}_{2}{ }^{1} \text { ) }=\text { ca. } 1,0 \text {. }
$$

Es war zunächst der Einfluss dieser Salze allein, und zwar in demselben Konzentrationsverhältnis, wie sie im Seewasser vorhanden sind, zu untersuchen.

Die möglichen Variationen und Kombinationen dieser Salze sind nun folgende:

II. Binäre Lösungen.

$$
\begin{aligned}
& \mathrm{NaCl}+\mathrm{KCl}, \\
& \mathrm{NaCl}+\mathrm{CaCl}_{2}, \\
& \mathrm{NaCl}+\mathrm{MgSO}_{4}, \\
& \mathrm{NaCl}+\mathrm{MgCl}_{2} .
\end{aligned} \quad\left\{\begin{array}{l}
\mathrm{KCl}+\mathrm{CaCl}_{2}, \\
\mathrm{KCl}+\mathrm{MgSO}_{4}, \\
\mathrm{KCl}+\mathrm{MgCl}_{2}, \\
\mathrm{CaCl}_{2}+\mathrm{MgSO}_{4}, \\
\mathrm{CaCl}_{2}+\mathrm{MgCl}_{2}, \\
\mathrm{MgSO}_{4}+\mathrm{MgCl}_{2} .
\end{array}\right.
$$

1) Die im Gegensatz zu den sehr konstanten relativen Mengen der anderen Salze schwankende Quantität von $\mathrm{CaCl}_{2}$ beträgt nach den Untersuchungen von L o eb usw. für Oaklandwasser ca. 1,0 Molekularteil. 


$$
\begin{gathered}
\text { III. Ternäre Lösungen. } \\
\mathrm{NaCl}+\mathrm{KCl}+\mathrm{CaCl}_{2}, \\
\mathrm{NaCl}+\mathrm{KCl}+\mathrm{MgSO}_{4}, \\
\mathrm{NaCl}+\mathrm{KCl}+\mathrm{MgCl}_{2}, \\
\mathrm{NaCl}+\mathrm{CaCl}_{2}+\mathrm{MgSO}_{4}, \\
\mathrm{NaCl}+\mathrm{CaCl}_{2}+\mathrm{MgCl}_{2}, \\
\mathrm{NaCl}+\mathrm{MgSO}_{4}+\mathrm{MgCl}_{2} . \\
\mathrm{IV} . \mathrm{Quaternäre} \mathrm{Lösungen.} \\
\mathrm{NaCl}+\mathrm{KCl}+\mathrm{CaCl}_{2}+\mathrm{MgSO}_{4}, \\
\mathrm{NaCl}+\mathrm{KCl}+\mathrm{CaCl}_{2}+\mathrm{MgCl}_{2}, \\
\mathrm{NaCl}+\mathrm{KCl}+\mathrm{MgSO}_{4}+\mathrm{MgCl}_{2}, \\
\mathrm{NaCl}+\mathrm{CaCl}+\mathrm{MgSO}_{4}+\mathrm{MgCl}_{2} . \\
\mathrm{V} . \mathrm{van} \text { 't } \mathrm{Hoff} \text { 'sehe } \mathrm{L}_{0} \text { ö ung. } \\
\mathrm{NaCl}+\mathrm{KCl}+\mathrm{CaCl}_{2}+\mathrm{MgSO}_{4}+\mathrm{MgCl}_{2} .
\end{gathered}
$$

\section{I.}

Von den Lösungen, die nur ein Salz enthielten, war natürlich $\mathrm{NaCl}$ am interessantesten. Die Kurve, welche die Mittelwerte von Männchen und Weibchen ${ }^{1}$ ) darstellt, zeigt den toxischen Effekt reiner Kochsalzlösung.

Die Kurve besitzt zunächst eine ähnliche Gestalt wie die des Seewassers. Auch hier ist ein plötzlicher, steiler Abfall, der bei Weiterführung der Messungen auf verdünntere Lösungen noch deutlicher zuTage treten würde, festzustellen. Bei den höheren Konzentrationen verläuft die Kurve ebenfalls langsam absteigend und sich assymptotisch der Abszissenachse nähernd. Ein bemerkenswerter Unterschied aber zur Seewasserkurve besteht darin, dass die Lebenszeiten in Summa einen bedeutend niedrigeren Wert haben, dass also eine reine NaCl-Lösung bei weitem giftiger ist als das Gemisch von Salzen, welches das Seewasser darstellt. Diese starke Giftigkeit des reinen NaCl fällt, wird besonders deutlich bei Betrachtung. z. B. von Figur 2, in welcher unter die Seewasserkurven noch die des reinen $\mathrm{NaCl}$, und zwar in der Konzentration, in der es in dem entsprechenden, mit der Kon-

1) Der Raumersparnis und der Deutlichkeit halber wurden die Einzelwerte für Männchen und Weibchen nur in Tabellenform gegeben. 


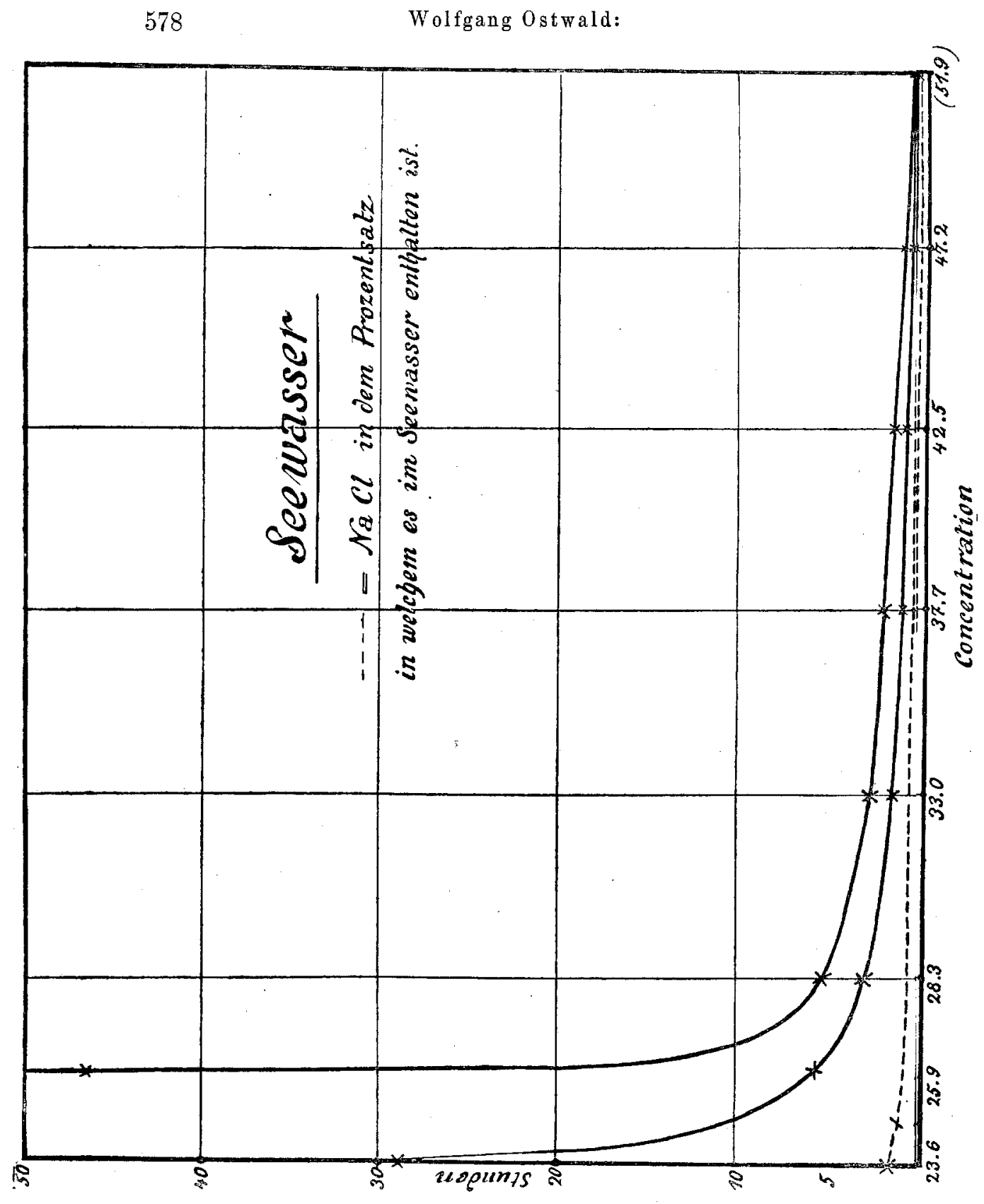

Fig. 2.

zentrationszahl versehenem Seewasser enthalten ist. Trotzdem die $\mathrm{NaCl-Lösung} \mathrm{also} \mathrm{an} \mathrm{und} \mathrm{für} \mathrm{sich} \mathrm{weniger} \mathrm{konzentriert} \mathrm{ist} \mathrm{als} \mathrm{das} \mathrm{ent-}$ sprechende Seewasser, hat sic doch eine bedeutend grössere Giftigkeit. 
In gewissem Sinne entspricht auch dies dem Verhalten von Seewasseramphipoden in verdünnteren Lösungen, indem J. Lo eb ${ }^{1}$ ) fand, dass sich eine mit Seewasser isosmotische NaCl-Lösung ungefähr ebenso giftig erwies wie destilliertes Wasser.

Die ausführlichen Zahlen sind in folgender Tabelle wiedergegeben.

$$
\text { Tabelle 2. } \mathrm{NaCl}_{x}
$$

\begin{tabular}{c|c|c|c}
\hline $\begin{array}{c}\text { Konzentration } \\
\text { in \%,000 }\end{array}$ & $\begin{array}{c}\text { Lebenzeit des } \\
\text { Weibchens } \\
\text { in Minuten }\end{array}$ & $\begin{array}{c}\text { Lebenszeit des } \\
\text { Männchens } \\
\text { in Minuten }\end{array}$ & Mittlere Lebenszeit \\
\hline 43,8 & 27 & 46 & 36,5 \\
41,6 & 33 & 52 & 42,5 \\
37,2 & 39 & 67 & 53,0 \\
32,8 & 42 & 86 & 57,0 \\
28,4 & 51 & 95 & 66,0 \\
24,0 & 57 & 104 & 76,0 \\
21,8 & 67 & - & 85,5 \\
$\left.15,3^{2}\right)$ & - & & 243,0
\end{tabular}

Es ergibt sich theoretisch aus dieser im Vergleich zu Seewasser (siehe Fig. 2) so bedeutend grösseren Giftigkeit von reinem $\mathrm{NaCl}$, dass das Hinzufügen der anderen Salze $\left(\mathrm{KCl}, \mathrm{CaCl}_{2}, \mathrm{MgCl}_{2}\right.$ und $\mathrm{MgSO}_{4}$ ) eine kompensatorische oder eine dem $\mathrm{NaCl}$ antagonistische Wirkung haben muss. Welcher Art diese antagonistische Wirkung ist, lässt sich zunächst nicht sagen; einige theoretische Erörterungen dieses Punktes sollen später folgen. Was den Charakter der weiteren Messungen aber anbetrifft, so folgt aus dem geschilderten Verhalten, dass er im allgemeinen in einem $A$ ufbau der einzelnen Salzvariationen und -kombinationen, deren spezifisches Verhalten dabei sich nicht voraussagen lässt, bestehen wird.

Was nun Einzellösungen von den übrigen Salzen in destilliertem Wasser anbetrifft, so war angesichts ihrer geringen relativen Quantität von vornherein zu erwarten, dass ihr Einfluss sich nicht sehr bemerkenswert zeigen würde. Es wurde dementsprechend von jedem Salz nur eine Messung gemacht, und zwar wurden Lösungen be-

1) J. L o e b, Pflüger's Archiv Bd. 97 S. 394 ff. 1903.

2) Die Bestimmungen der Giftigkeit derartig verdünnter Lösungen wurden am Schlusse der Untersuchung gemacht, als das Material sehr knapp wurde. Ich erhielt dementsprechend nicht genügend Pärchen mebr und konnte also nur den Mittelwert bestimmen. 
nutzt, welche etwas mehr Salz enthielten, als doppeltkonzentriertem Seewasser entsprechen würde.

Es ergab sich:

$$
\text { Tabelle } 3 \text {. }
$$

KCl. $100 \mathrm{ccm}$ dest. Wassers $+4,4 \mathrm{ccm}^{3} / \mathrm{s} \mathrm{n}$. KCl.

$$
\begin{aligned}
& \text { Weibchen . . . } 216 \text { Minuten }=c a_{0} 3^{1 / 2} \text { Stunden } \\
& \text { Männchen . . . . . } 354 \text { Minuten }=\text { ca. } 6 \text { Stunden } \\
& \mathrm{MgCl}_{2} \cdot 100 \mathrm{ccm} \text { dest. Wassers }+15,6 \mathrm{~cm}{ }^{3 / 8} \mathrm{n} . \mathrm{MgCl}_{2} \text {. } \\
& \text { Weibchen . . . . . . . . 19-20 Stunden } \\
& \text { Männchen . . . . . . . . . . 46-48 Stunden } \\
& \mathrm{MgSO}_{4} .100 \mathrm{ccm} \text { dest. Wassers }+7,8 \mathrm{ccm}{ }^{3 / 8} \mathrm{n} . \mathrm{MgSO}_{4} \text {. } \\
& \text { Weibchen . . . . . . . . 26-28 Stunden } \\
& \text { Männchen .......... 70-76 Stunden } \\
& \mathrm{CaCl}_{2} \cdot 100 \mathrm{~cm} \text { dest. Wassers }+2 \mathrm{~cm}{ }^{3} / 8 \mathrm{n} . \mathrm{CaCl}_{2} \text {. } \\
& \text { Weibchen........... ca. } 3 \text { Tage } \\
& \text { Männchen. . . . . . . . . ca. 14-16 Tage. }
\end{aligned}
$$

Die Reihenfolge der Giftigkeit der einzelnen Salze in der relativen Seewasserkonzentration ist also $\mathrm{KCl}, \mathrm{MgCl}_{2}, \mathrm{MgSO}_{4}$ und $\mathrm{CaCl}_{2} . \mathrm{KCl}+\mathrm{CaCl}_{2}$ sind dabei die Extreme, indem $\mathrm{KCl}$ in drei bis sechs Stunden tödlich wirkt, während das nicht halb so konzentrierte $\mathrm{CaCl}_{2}$ im Gegensatz hierzu seinen schädlichen Einfluss beim Weibchen nach ca. drei Tagen, beim Männchen erst nach über zwei Wochen zeigt.

\section{Binäre Lösungen.}

Von binären Lösungen wurden nur die Kombinationen mit $\mathrm{NaCl}$ als besonders wichtig untersucht. Die übrigen Kombinationen der nur in kleinen Quantitäten im Seewasser vorhandenen Salze (siehe oben S. 576) erschienen entsprechend den im vorigen Abschnitt beschriebenen Einzelversuchen zu schwach, um bestimmtere, speziell graphisch darstellbare Resultate zu ergeben. Nichtsdestoweniger sind natürlich ergänzende Beobachtungen wünschenswert.

Die Kurven auf Taf. III veranschaulichen die Ergebnisse. Es ist zu der graphischen Darstellung zu bemerken, dass der Deutlichkeit wegen die Mittelwertskurve jeder Lösung um 60 Minuteneinheiten nach oben verschoben worden ist, so dass, wie die an die Kurven geschriebenen Zahlen es zeigen, von den Ordinaten $0,60,120$, 180 usw. Einheiten abgezogen werden müssen, um die absoluten Werte der Lebensdauer zu erhalten. 
Versuche über die Giftigkeit des Seewassers für Süsswassertiere.

Die genauen Zahlen sind:

Tabelle 4. $\mathrm{NaCl}+\mathrm{MgCl}_{2}$.

\begin{tabular}{c|c|c|c}
\hline Konzentration & $\begin{array}{c}\text { Lebenszeit des } \\
\text { Weibchens } \\
\text { in Minuten }\end{array}$ & $\begin{array}{c}\text { Lebenszeit des } \\
\text { Männchens } \\
\text { in Minuten }\end{array}$ & Mittlere Lebenszeit \\
\hline$\left.H^{1}\right)$ & 25 & 46 & 31,5 \\
$E$ & 30 & 49 & 39,5 \\
$C$ & 40 & 73 & 56,5 \\
$B$ & 52 & - & 171,5 \\
$A$ & - & - & 235,0
\end{tabular}

Tabelle 5. $\mathrm{NaCl}+\mathrm{MgSO}_{4}$.

\begin{tabular}{c|c|c|c}
\hline Konzentration & $\begin{array}{c}\text { Lebenzzeit des } \\
\text { Weibchens } \\
\text { in Minuten }\end{array}$ & $\begin{array}{c}\text { Lebenszeit des } \\
\text { Männchens } \\
\text { in Minuten }\end{array}$ & Mittlere Lebenszeit \\
\hline$H$ & 33 & 58 & 43 \\
$E$ & 37 & 63 & 50 \\
$C$ & 49 & 98 & 73,5 \\
$B$ & 68 & 108 & 86 \\
$A$ & - & - & 273
\end{tabular}

Tabelle 6. $\mathrm{NaCl}+\mathrm{CaCl}_{2}$.

\begin{tabular}{c|c|c|c}
\hline Konzentration & $\begin{array}{c}\text { Lebenszeit des } \\
\text { Weibchens } \\
\text { in Minuten }\end{array}$ & $\begin{array}{c}\text { Lebenszeit des } \\
\text { Männchens } \\
\text { in Minuten }\end{array}$ & Mittlere Lebenszeit \\
\hline $\boldsymbol{H}$ & 28 & 37 & 32,5 \\
$\boldsymbol{E}$ & 34 & 59 & 46,5 \\
$\boldsymbol{B}$ & 43 & 71 & 57 \\
$\boldsymbol{A}$ & 82 & 108 & 85 \\
& - & - & 339
\end{tabular}

Tabelle 7. $\mathrm{NaCl}+\mathrm{KCl}$.

\begin{tabular}{c|c|c|c}
\hline Konzentration & $\begin{array}{c}\text { Lebenszeit des } \\
\text { Weibchens } \\
\text { in Minuten }\end{array}$ & $\begin{array}{c}\text { Lebenszeit des } \\
\text { Männchens } \\
\text { in Minuten }\end{array}$ & Mittlere Lebenszeit \\
\hline$H$ & 25 & 41 & 33 \\
$E$ & 36 & 49 & 43 \\
$C$ & 53 & 78 & 65,5 \\
$B$ & 64 & 129 & 96,5 \\
$A$ & - & - & 368
\end{tabular}

1) Die Buchstaben sollen bezeichnen, dass zu den entsprechen̆d benannten $\mathrm{NaCl}$-Lösungen die zugeordneten Mengen der anderen Salze zugesetzt wurden, z. B. zu $100 \mathrm{~cm} 3 / 8$ norm. $\mathrm{NaCl} 7,8 \mathrm{~cm} 3 / 8$ norm. $\mathrm{MgCl}_{2}, 2,1 \mathrm{~cm} 3 / 8$ norm. KCl usw., zu $100 \mathrm{~cm} 6 / 8$ norm. $\mathrm{NaCl} 7,8 \mathrm{ccm}^{6} / 8$ norm. $\mathrm{MgCl}, 2,1 \mathrm{~cm} 6 / 8$ norm. $\mathrm{KCl}$ usw. 
Was nun die Gestalt der binären Lösungskurven anbetrifft, so zeigen sie zunächst ebenfalls den allgemeinen Charakter der bisher beschriebenen Kurven: steiler Abfall mit fast geradlinigem und asymptotischem Verlauf bei höheren Konzentrationen. Die Unterschiede zur reinen NaCl-Lösung bestehen im folgenden:

Zunächst verhalten sich die vier binäreu Lösungskurven nicht ganz gleich zueinander; speziell nimmt die Kurve für $\mathrm{NaCl}+\mathrm{MgCl}_{2}$ eine besondere Stellung im Vergleich $z u$ reiner NaCl-Lösung ein. Es zeigte sich, dass $\mathrm{NaCl}+\mathrm{MgCl}_{2}$ in dem Gewichtsverbältnis, in dem beide im Seewasser enthalten sind, noch etwas giftiger ist als $\mathrm{NaCl}$ allein. Und zwar besteht, wie ein Vergleich der entsprechenden Zahlen zeigt, dieser Unterschied für die ganze Kurve, d. h. für alle Konzentrationen. Wennschon dieser Unterschied nicht viel beträgt, so macht die Konstanz desselben für die ganze Kurve es sicher, dass es sich nicht um Versuchs- oder Beobachtungsfehler handelt.

Sehr bemerkenswert verhalten sich indessen die Kurven der Gemische mit den anderen drei Salzen. Alle drei besitzen nämlich höhere absolute Werte, d. b. zeigen an, dass sie eine geringere Giftigkeit besitzen als $\mathrm{NaCl}$ allein. Dies bedeutet mit anderen Worten, dass diese drei Salze $\left(\mathrm{KCl}, \mathrm{CaCl}_{2}\right.$ und $\left.\mathrm{MgSO}_{4}\right)$ antagonistisch auf die Giftigkeit des NaCl wirken.

Die Reihenfolge in bezug auf diese Kompensationsfähigkeit ist $\mathrm{MgSO}_{4}, \mathrm{CaCl}_{2}$ und $\mathrm{KCl}$. Von diesen zeigt sich $\mathrm{KCl}$ mit 6 Stunden 8 Minuten für die Konzentration $A$ als das günstigste Salz. Es ist dies eine sehr verwunderliche Tatsache, darum nämlich, weil wir sahen, dass als $\mathrm{Einzellösung} \mathrm{KCl}$, obgleich neben $\mathrm{CaCl}_{2}$ nur in sehr kleinem Prozentsatz im Seewasser enthalten, bei weiten a m giftigsten von allen Salzen wirkt.

Während sich nun $\mathrm{KCl}$ und $\mathrm{CaCl}_{2}$ in Kombination mit $\mathrm{NaCl}$ bis auf den bezeichneten Unterschied selır ähnlich verhalten (siehe die Kurven), nimmt $\mathrm{MgSO}_{4}$ eine etwas gesonderte Stellung ein. Auffällig . sind zunächst die sehr hohen Zahlen bei den hohen Konzentrationen, welche bis zur Konzentration $C$ die böchsten von allen vier Kurven sind und den entsprechenden Werten der Seewasserkurve fast, gleichkommen. Dann besteht eine Ähnlichkeit mit der $\mathrm{MgCl}_{2}$-Kurve insofern, als in diesen beiden der $\mathrm{Abfall}$ der Kurve im Vergleich $\mathrm{zu}$ den $\mathrm{KCl}$ - und $\mathrm{CaCl}_{2}$-Kurven ähnlich wie auch bei reinem $\mathrm{NaCl}$ deutlich allmählicher oder langsamer erfolgt. 
Es ist nur ein anderer Ausdruck für die besprochenen Tatsachen, wenn wir den Unterschied zwischen den Konbinationen $\mathrm{NaCl}+\mathrm{KCl}, \mathrm{NaCl}+\mathrm{CaCl}_{2}, \mathrm{NaCl}+\mathrm{MgSO}_{4}$ sowie anderseits $\mathrm{NaCl}+$ $\mathrm{MgCl}_{2}$ und reiner NaCl-Lösung so präzisieren, dass in den ersteren Lösungen der Abfall der Kurven später, bei einer böheren Konzentration beginnt als bei den zu zweit genannten Lösungen.

Es sei gleich hier bemerkt, dass zusammen mit dem Verhalten der reinen NaCl-Lösung diese Versuche deutlich zeigen, dass die Giftwirkungen des Seewassers auf Süsswassertiere durchaus nicht rein "physikalischer", d. i. osmotischer Natur sind. Denn falls sie dies wären, dürfte zunächst nicht die reine NaCl-Lösung so bedeutend giftiger sein als ihre Kombinatiou mit den übrigen vier Salzen zum Seewasser; es müsste fernerhin das Hinzufügen der übrigen Salze diese Giftigkeit steigern, und zwaŕ im Verhältnis ihrer relativen Konzentration, - eine Forderung, die indessen, wie wir sehen, keineswegs bestätigt wird:

III. Ternäre Lösungen.

Die Resultate der Versuche mit ternären Lösungen sind graphisch auf Taf. IV dargestellt. Die gewonnenen ausführlichen Zahlen sind in folgenden Tabellen enthalten:

Tabelle 8. $\mathbf{N a C l}+\mathrm{MgCl}_{2}+\mathrm{MgSO}_{4}$.

\begin{tabular}{c|c|c|c}
\hline Konzentration & $\begin{array}{c}\text { Lebenszeit des } \\
\text { Weibchens } \\
\text { in Minuten }\end{array}$ & $\begin{array}{c}\text { Lebenszeit des } \\
\text { Männchens } \\
\text { in Minuten }\end{array}$ & $\begin{array}{c}\text { Mittlere } \\
\text { Lebenszeit }\end{array}$ \\
\hline$H$ & 26 & 47 & 36,5 \\
$E$ & 38 & 59 & 48,5 \\
$B$ & 50 & 98 & 74,0 \\
$A$ & - & - & ca. 5 Stunden
\end{tabular}

Tabelle 9. $\mathrm{NaCl}+\mathrm{CaCl}_{2}+\mathrm{MgSO}_{4}$

\begin{tabular}{c|c|c|c}
\hline Konzentration & $\begin{array}{c}\text { Lebenszeit des } \\
\text { Weibchens } \\
\text { in Minuten }\end{array}$ & $\begin{array}{c}\text { Lebenszeit des } \\
\text { Männchens } \\
\text { in Minuten }\end{array}$ & $\begin{array}{c}\text { Mittlere } \\
\text { Lebenszeit }\end{array}$ \\
\hline$H$ & 27 & 48 & 37,5 \\
$E$ & 43 & 61 & 52,0 \\
$B$ & 55 & 124 & 89,5 \\
$A$ & - & - & ca. 6 Stunden
\end{tabular}


Tabelle 10. $\mathrm{NaCl}+\mathrm{CaCl}_{2}+\mathrm{MgCl}_{2}$.

\begin{tabular}{c|c|c|c}
\hline Konzentration & $\begin{array}{c}\text { Lebenszeit des } \\
\text { Weibchens } \\
\text { in Minuten }\end{array}$ & $\begin{array}{c}\text { Lebenszeit des } \\
\text { Männchens } \\
\text { in Minuten }\end{array}$ & $\begin{array}{c}\text { Mittlere } \\
\text { Lebenszeit }\end{array}$ \\
\hline$H$ & 22 & 41 & 31,5 \\
$E$ & 41 & 60 & 51,5 \\
$B$ & 53 & 79 & 66,0 \\
$A$ & - & - & ca. 5 Stunden
\end{tabular}

Tabelle 11. $\mathrm{NaCl}+\mathrm{KCl}+\mathrm{MgSO}_{4^{*}}$

\begin{tabular}{c|c|c|c}
\hline Konzentration & $\begin{array}{c}\text { Lebenszeit des } \\
\text { Weibchens } \\
\text { in Minuten }\end{array}$ & $\begin{array}{c}\text { Lebenszeit des } \\
\text { Männchens } \\
\text { in Minuten }\end{array}$ & $\begin{array}{c}\text { Mittlere } \\
\text { Lebenszeit }\end{array}$ \\
\hline$H$ & 38 & 47 & 42,5 \\
$E$ & 42 & 74 & 58,0 \\
$\boldsymbol{B}$ & 80 & 131 & 105,5 \\
$\boldsymbol{A}$ & - & - & ca. 13 Stunden
\end{tabular}

Tabelle 12. $\mathrm{NaCl}+\mathrm{KCl}+\mathrm{MgCl}_{2}$.

\begin{tabular}{c|c|c|c}
\hline Konzentration & $\begin{array}{c}\text { Lebenszeit des } \\
\text { Weibchens } \\
\text { in Minuten }\end{array}$ & $\begin{array}{c}\text { Lebenszeit des } \\
\text { Männchens } \\
\text { in Minuten }\end{array}$ & $\begin{array}{c}\text { Mittlere } \\
\text { Lebenszeit }\end{array}$ \\
\hline$H$ & 38 & 46 & 42 \\
$E$ & 62 & 73 & 57,5 \\
$\boldsymbol{B}$ & 53 & - & ca. 5 Stunden
\end{tabular}

Tabelle 13. $\mathrm{NaCl}+\mathrm{KCl}+\mathrm{CaCl}_{2}$.

\begin{tabular}{c|c|c|c}
\hline Konzentration & $\begin{array}{c}\text { Lebenszeit des } \\
\text { Weibchens } \\
\text { in Minuten }\end{array}$ & $\begin{array}{c}\text { Lebenszeit des } \\
\text { Männchens } \\
\text { in Minuten }\end{array}$ & $\begin{array}{c}\text { Mittlere } \\
\text { Lebenszeit }\end{array}$ \\
\hline$H$ & 39 & 57 & 48 \\
$E$ & 44 & 94 & 69 \\
$\boldsymbol{A}$ & 72 & - & ca. 19 Stunden
\end{tabular}

Gemeinsam mit den bisher besprochenen Kurven sind für die Darstellungen der Giftigkeit der ternären Lösungen wiederum der steile Abfall und der darauffolgende fast geradlinige und asymp- 
totische Verlauf bei höheren Konzentrationen. Verschieden von ihnen jedoch ist der Umstand, dass sämtliche ternäre Lösungen einen steileren und späteren Abfall haben, dass also, mit anderen Worten, alle ternären Lösungen weniger giftig sind als $\mathrm{NaCl}$ und die binären Gemische.

Im speziellen ist die Wirkung der einzelnen Kombinationen ziemlich kompliziert.

Die beste Kombination - d. h. diejenige, welche am wenigsten giftig ist und sich so dem Seewasser am meisten nähert - ist $\mathrm{NaCl}+\mathrm{KCl}+\mathrm{CaCl}_{2}$. Interessant ist, dass bei den bohen Konzentrationen und entsprechend kurzen Lebenszeiten die entsprechenden Werte die Seewasserkurve nicht nur erreichen, sondern deutlich überschreiten (54 zu 47 für das Mittel zwischen Männchen und Weibchen). Ferner ist sehr bemerkenswert der ganz ausserordentlich steile Abfall dieser Kurve, ferner der späte, d. h. erst bei relativ hohen Konzentrationen erfolgende Eintritt desselben.

Dieser Mischung zunächst kommt $\mathrm{NaCl}+\mathrm{KCl}+\mathrm{MgSO}_{4}$. Auch hier macht sich neben dem begünstigenden Einfluss auch die bei den binären Lösungen festzustellende Tendenz des $\mathrm{MgSO}_{4}$, die Steilheit des Abfalls der Kurve zu vermindern, in der langsam sich biegenden Gestalt geltend (vergleiche insbesondere die Strecken $\overline{B E}$ der verschiedenen Kurven). Auch hier beträgt die Giftigkeit der Lösung von einer relativen Konzentration, wie sie dem Seewasser entspricht, ungefähr ebensoviel wie die des Seewassers selbst (ca. 47 Minuten).

Die dritte Stelle nimmt die Kombination $\mathrm{NaCl}+\mathrm{CaCl}_{2}+$ $\mathrm{MgSO}_{4}$ ein. Auch in dieser Kurve macht sich der spezifische Einfluss des $\mathrm{MgSO}_{4}$, der in einer Abschwächung und Verlangsamung des Abfalls besteht, deutlich sichtbar, insbesondere wenn man die analoge, nur mit $\mathrm{MgCl}_{2}$ anstatt $\mathrm{MgSO}_{4}$ zusammengesetzte Kurve betrachtet, speziell zwischen $B$ und $E$. Die andere Eigentümlichkeit des $\mathrm{MgSO}_{4}$, die hohen Werte bei den starken Konzentrationen, ist in einer dreifachen Lösung fast verschwunden. Während der H-Wert noch um eine Minute grösser ist als der entsprechende Wert der Kombination $\mathrm{NaCl}+\mathrm{KCl}+\mathrm{MgCl}_{2}$, ist er schon um fünf Minuten kleiner als in der Lösung $\mathrm{NaCl}+\mathrm{KCl}+\mathrm{CaCl}_{2}$.

Als eng zusammengehörig erweisen sich die übrigen drei Kurven, namentlich bei den niedrigeren Konzentrationen. Alle drei sind hier noch relativ stark giftig, indem die Lebenszeit z. B. bei der Kon- 
zentration $A$ in merkwürdiger Übereinstimmung für alle drei Kombinationen nur zirka fünf Stunden beträgt. Es ist interessant, zu sehen, dass dieses gleichartige Verhalten allem Anschein nach nur dem Umstande, dass $\mathrm{MgCl}_{2}$ jedesmal einer der in der Kombination enthaltenen Bestandteile ist, zuzuschreiben ist. Wie schon bei den binären Lösungen so zeigt sich auch hier ausserordentlich deutlich die starke, die Wirkungen des $\mathrm{NaCl}$ unterstützende Giftigkeit des $\mathrm{MgCl}_{2}$. Die Reihenfolge der Lösungen, welche $\mathrm{MgCl}_{2}$ enthalten, ist entsprechend den anderen im Gemisch enthaltenen Salzen:

$$
\begin{aligned}
& \mathrm{NaCl}+\mathrm{KCl}+\mathrm{MgCl}_{2}, \text { als die beste Kombination, } \\
& \mathrm{NaCl}+\mathrm{CaCl}_{2}+\mathrm{MgCl}_{2}, \\
& \mathrm{NaCl}+\mathrm{MgSO}_{4}+\mathrm{MgCl}_{2} .
\end{aligned}
$$

In der letzteren Kombination macht sich wieder in der langsam gebogenen Gestalt der Kurve sowie in den relativ grossen Lebenszeiten bei hohen Konzentrationen der spezifische Finfluss des $\mathrm{MgSO}_{4}$ geltend.

Schon jetzt schält sich eine gewisse Charakteristik und Spezifizität der Einzelwirkungen der genannten, zum Teil mit $\mathrm{NaCl}$ gleichsinnig $\left(\mathrm{MgCl}_{2}\right)$, zum Teil aber antagonistiseh wirkenden Salze heraus. Indessen soll zweckmässigerweise erst am Schluss der Versuchsschilderungen etwas näher hierauf eingegangen werden.

\section{Quaternäre Lösungen.}

Taf. V veranschaulicht die Versuche mit quaternären Lösungen. Die genauen und ausführlichen Zahlen geben die folgenden Tabellen:

Tabelle 14. $\mathrm{NaCl}+\mathrm{CaCl}_{2}+\mathrm{MgCl}_{2}+\mathrm{MgSO}_{4}$.

\begin{tabular}{c|c|c|c}
\hline Konzentration & $\begin{array}{c}\text { Lebenszeit des } \\
\text { Weibchens } \\
\text { in Minuten }\end{array}$ & $\begin{array}{c}\text { Lebenszeit des } \\
\text { Männchens } \\
\text { in Minuten }\end{array}$ & $\begin{array}{c}\text { Mittlere } \\
\text { Lebenszeit }\end{array}$ \\
\hline$H$ & 29 & 37 & 33 \\
$E$ & 32 & 53 & 42,5 \\
$B$ & 66 & - & ca. $51 / 2$ Stunden \\
$A$ & - & &
\end{tabular}

Die Kurven lassen sich wiederum in zwei Gruppen teilen, in solche, welche Kombinationen mit $\mathrm{MgCl}_{2}$ darstellen, und solche ohne dies Salz (in diesem Falle nur eine Kombination). Und zwar nimmt diese letztere Kurve. die Kombination $\mathrm{NaCl}+\mathrm{KCl}+$ 
$\mathrm{CaCl}_{2}+\mathrm{MgSO}_{4}$ darstellend, in ganz isoliert stehendem Masse die erste Stelle in bezug auf Verminderung der Giftigkeit und Annäherung an die Seewasserkurve ein. Die anderen drei $\mathrm{MgCl}_{2}$ enthaltenden Kombinationen gehen kaum über die entsprechenden ternären Lösungen hinaus $\left(5^{1 / 2} \quad\right.$ zu $\quad 5$ Stunden $)$, zeigen aber wiederum sehr charakteristisch den dominierenden Charakter der $\mathrm{MgCl}_{2}$-Wirkung, dadurch, dass sie alle drei sehr auffälligerweise dieselbe Zahl ( $5^{1 / 2}$ Stunden) für die Konzentration $A$ ergeben.

Tabelle 15. $\mathrm{NaCl}+\mathrm{KCl}+\mathrm{MgCl}_{2}+\mathrm{MgSO}_{4}$.

\begin{tabular}{c|c|c|c}
\hline \hline Konzentration & $\begin{array}{c}\text { Lebenszeit des } \\
\text { Weibchens } \\
\text { in Minuten }\end{array}$ & $\begin{array}{c}\text { Lebenszeit des } \\
\text { Männchens } \\
\text { in Minuten }\end{array}$ & $\begin{array}{c}\text { Mittlere } \\
\text { Lebenszeit }\end{array}$ \\
\hline$H$ & 29 & 41 & 35 \\
$E$ & 37 & 61 & 49 \\
$B$ & 68 & 92 & 80 \\
$A$ & - & - & cad. 5 St/2 Stunden
\end{tabular}

Tabelle 16. $\mathrm{NaCl}+\mathrm{KCl}+\mathrm{CaCl}_{2}+\mathrm{MgSO}_{4}$.

\begin{tabular}{c|c|c|c}
\hline Konzentration & $\begin{array}{c}\text { Lebenszeit des } \\
\text { Weibchens } \\
\text { in Minuten }\end{array}$ & $\begin{array}{c}\text { Lebenszeit des } \\
\text { Männchens } \\
\text { in Minuten }\end{array}$ & $\begin{array}{c}\text { Mittlere } \\
\text { Lebenszeit }\end{array}$ \\
\hline$H$ & 30 & 42 & 36 \\
$E$ & 35 & 58 & 46,5 \\
$B$ & 74 & 110 & 92 \\
$A$ & - & - & ca. 34 Stunden
\end{tabular}

Tabelle 17. $\mathrm{NaCl}+\mathbf{K C l}+\mathrm{CaCl}_{2}+\mathrm{MgCl}_{2}$.

\begin{tabular}{c|c|c|c}
\hline Konzentration & $\begin{array}{c}\text { Lebenszeit des } \\
\text { Weibchens } \\
\text { in Minuten }\end{array}$ & $\begin{array}{c}\text { Lebenszeit des } \\
\text { Männchens } \\
\text { in Minuten }\end{array}$ & $\begin{array}{c}\text { Mittlere } \\
\text { Lebenszeit }\end{array}$ \\
\hline$H$ & 28 & 42 & 35 \\
$E$ & 40 & 52 & 46 \\
$B$ & 47 & 89 & 86 \\
$A$ & - & - & ca. 51/2 Stunden
\end{tabular}

Auch der charakteristische Einfluss des $\mathrm{MgSO}_{4}$ ist in den Kurven wiederzuerkennen, mit Ausnahme allerdings der Kombination $\mathrm{NaCl}+\mathrm{KCl}+\mathrm{CaCl}_{2}+\mathrm{MgSO}_{4}$, in welcher der lebenverlängernde 
Einfluss des $\mathrm{KCl}+\mathrm{CaCl}_{2}$ so stark ist, dass die $\mathrm{MgSO}_{4}$-Wirkung von ihm superponiert wird. Sehr deutlich ist dieser Effekt des $\mathrm{MgSO}_{4}$ aber beim Vergleich der anderen drei Kurven zu ersehen, namentlich bei Betrachtung der Steilheit der Strecken $\overline{A B}$ (90 bis $5^{1 / 2}, 80$ bis $5^{1 / 2}$ und in der $\mathrm{MgSO}_{4}$-freien Lösung 68 bis $5^{1 / 2}$ ).

Was das Verhalten bei den höheren Konzentrationen anbetrifft, so ist speziell im Vergleich zu den ternären Lösungen zweierlei zu beobachten: erstens ein allgemeines Sinken der Lebenszeiten (von max. 48 auf max. 36 Minuten für die Konzentration $H$, von max. 69 auf max. 49 für die Konzentration $E$ usw.); zweitens eine Annäherung der Werte für alle Kombinationen respektive das Verlöschen der Spezifität der einzelnen Salzwirkungen (bei ternären Lösungen Extreme 32 und 48 Minnten, hier 33 und 36). Der erste Umstand ist von Interesse darum, weil hierdurch dieser Teil der Kurven im Gegensatz zu den zu hohen ternären Lösungen jetzt auf eine Höhe gebracht wird, die wiederum etwas zu niedrig ist im Vergleich zu den entsprechenden Werten des Seewassers, wennschon jetzt die positive Differenz mit dem Seewasser nicht so gross ist wie die negative bei den ternären Lösungen. Dieses oszillatorische Schwanken der Giftigkeit der konzentrierten Lösungen ist ein sehr sonderbares und unverständliches Verhalten.

In analoger Weise wie in den vorigen Abschnitten ist auch hier zu sagen, dass bei der optimalen Kurve der Abfall später sowie hier bedeutend steiler eintritt als in den anderen Lösungen.

\section{V. van't Hoff'sche Lösung.}

Diese Versuche beziehen sich auf ein Gemisch von allen fünf genannten Salzen in dem Verbältnis, in dem diese im Seewasser vorhanden sind, benutzen also ein bis auf den Mangel von Spuren von Phosphaten, Eisen, Schwefel usw. künstliches Seewasser. Die Resultate sind auf Taf. VI u. VII und in folgender Tabelle enthalten.

Tabelle 18. van't Hoff'sche Lösung.

\begin{tabular}{c|c|c|c}
\hline Konzentration & $\begin{array}{c}\text { Lebenszeit des } \\
\text { Weibchens } \\
\text { in Minuten }\end{array}$ & $\begin{array}{c}\text { Lebenszeit des } \\
\text { Männchens } \\
\text { in Minuten }\end{array}$ & $\begin{array}{c}\text { Mittlere } \\
\text { Lebenszeit }\end{array}$ \\
\hline$H$ & 38 & 48 & 43 \\
$E$ & 59 & 80 & 69,5 \\
$B$ & 78 & 119 & 98,5 \\
$A$ & - & - & ca. 96 Stunden \\
(4 Tage)
\end{tabular}


In der Tat ergeben diese Versuche, dass eine van't Hoff' sche Lösung im allgemeinen sich in bezug auf ihre Giftigkeit sehr ähnlich wie Seewasser von entsprechender Konzentration verbält. Dies wird veranschaulicht zunächst auf T'af. VII, ferner sollen hier noch die entsprechenden Zahlen in Tabellenform angegeben werden.

Tabelle 19.

\begin{tabular}{c|c|c}
\hline \hline Konzentration & $\begin{array}{c}\text { van't Hoff'sche } \\
\text { Lösung }\end{array}$ & Seewasser \\
\hline$H$ & 43 & 43 \\
$D S$ & 56 & 47 \\
$E$ & 70 & 60 \\
$B$ & 99 & 109 \\
$\left\{A^{1}\right)$ & ca. 4 Tage & weniger als $5-6$ Tage \\
$S$ & - & $5-6$ Tage \\
& &
\end{tabular}

Es ist hier zu bemerken, dass zum direkten Vergleich die meisten der Zahlen graphisch interpoliert wurden, - ein Verfahren, das unter Umständen etwas mehr Unsicherheit als direkte Messungen besitzt.

Sehr interessant und sonderbar ist der Umstand, dass aus diesen Messungen deutlich hervorgeht, dass in der van't $\mathrm{H}$ off'schen Lösung auch das $\mathrm{MgCl}_{2}$ eine verbessernde - und zwar, wie z. $\mathrm{E}$. Taf. VI zeigt, eine durch alle Konzentrationen hindurchgehende Rolle spielt, im vollständigsten Gegensatz zu allen anderen Lösungen, in denen es enthalten ist. Es läge nach diesen Resultaten nahe, zu erwarten, dass die optimale quaternäre Lösung $(\mathrm{NaCl}+$ $\mathrm{KCl}+\mathrm{CaCl}_{2}+\mathrm{MgSO}_{4}$ ) dem Seewasser am nächsten kommt; und dass das Hinzufügen von $\mathrm{MgCl}_{2}$ auch in diesem Falle die Giftigkeit wieder erhöhen, d. h. die Kurve herunterbringen würde. Tatsächlich ergaben aber, wie gezeigt, die Mèssungen das Gegenteil.

Was die Abweichungen anbetrifft, so ist zunächst nicht von der Hand zu weisen, dass sie in Anbetracht namentlich ihrer relativen Geringfügigkeit auf den unvermeidlichen Versuchsfehlern beruhen. Namentlich gilt dies für die Strecke $\overline{B H}$ der v an't Hoff'schen Lösungskurve, in welcher wegen Mangels an gleichem Material nur eine Messungsreihe (Punkt $E$ ), und diese auch nur an zwölf Individuen, gemacht werden konnte. Es ist sehr möglich und mir wahr-

1) $A$ und $S$ variieren nur um $0,2 \%$. 
scheinlich, dass bei genanerer und umfangreicherer Messung der Wert für Punkt $E$ sich niedriger erweist, damit aber auch den Unterschied der Kurven bei den höheren Konzentrationen, der ja nur auf dieser einen Messung beruht, aufhebt.

Anders liegen die Verhältnisse wahrscheinlich bei der Kurvenstrecke rechts und besonders links von Punkt $B$. Hier habe ich mehr und genanere Messungen anstellen können, so dass die Abweichung wohl nicht fehlerhaften und ungenügenden Versuchen zuzuschreiben sein wird. Sie besteht in einem deutlich, bei den geringeren Konzentrationen allmählich wieder verlaufenden Sinken der Kurve für van't Hoff' sche Lösung, d. h. in einem Giftigerwerden derselben. Dies zeigt sowohl ein Vergleich der Kurven als auch (vielleicht noch besser) Tabelle 19. Mir war diese Abweichung lange Zeit hindurch unverständlich, bis ich, wie ich glaube, ihren Grund in folgendem fand.

Wie oben erwähnt wurde, hatte das Seewasser, mit dem ich arbeitete, insbesondere aber das doppeltkonzentrierte und dementsprechend alle Mischungen mit demselben, eine merklich alkalische Reaktion. Im Gegensatz hierzu war meine van't Hoff'sche Lösung, - wie speziell aus ihrer Zusammensetzung von Salzen folgt, welche sämtlich messbare Azidität zeigten - eine Spur sauer. Nun ist aber anderseits bekannt, dass Säuren zu den stärksten physiologischen Giften gehören. Ich glaubte nun, dass die negative Abweichung der van't Hoff'schen Lösung diesem Umstande zuzuschreiben ist, und versuchte, durch den spurenweisen Zusatz von Alkali, speziell dem von J. Loe $b^{1}$ ) in ähnlichen Verhältnissen mit Erfolg angewandtem $\mathrm{Na}_{2} \mathrm{CO}_{3}$ (resp. dem sehr schnell alkalisch werdenden $\mathrm{NaHCO}_{3}$ ), die Werte der Seewasserkurve zu erreichen. Di€s gelang mir vollständig, und zwar untersuchte ich die Konzentration $B$ daraufhin.

Die Resultate waren je nach der Menge des zugesetzten Karbonats resp. Bikarbonats nicht ganz gleich. Am besten erwies sich in einer ziemlich grossen Reihe von Versuchen ein Zusatz von $3 \mathrm{ccm}$ Bikarbonat und $0,5 \mathrm{cem}$ Karbonat zu $100 \mathrm{ccm}^{3} / 8$ va n't $\mathrm{H}$ off'scher Lösung (der Konzentration $B$ entsprechend). Ich erhielt im ersten Falle:

$$
\text { Weibchen }=107 \text {, Männchen }=124 \text { Minuten }
$$

1) J. Loeb, Pflüger's Archiv Bd. 101 S. 340 ff. 1904. 
und im zweiten:

$$
\text { Weibchen }=110, \text { Männchen }=163 \text { Minuten, }
$$

in beiden Fällen also deutlich höhere Werte als bei saurer van't Hoff'scher Lösung. Die Mittelwerte hiervon sind 110 und 137, sind also im Vergleich zu dem entsprechenden Werte der Seewasserkurve, der ca. 110 beträgt, noch etwas höher.

Leider habe ich aus erwähnten Gründen keine weiteren Versuche auch für andere Konzentrationen ausfübren können. Aus diesen hätte sich unter anderem wahrscheinlich ergeben, für den Fall, dass unserer Vermutung, entsprechend das Steigen der van 't $\mathrm{H}$ off' schen Kurve über die Seewasserkurve auf einem Versuchsfehler beruht, dass die Alkalinität der Lösung in Anbetracht ihrer sonstigen starken Giftigkeit keine so wichtige Rolle spielt wie bei den verdünnteren Lösungen, bei welchen eine längere Zeit bis zum Tode verfliesst. In analoger Weise wäre es aber auch möglich, dass das allmähliche Abnehmen und scheinbare Verschwinden dieser Differenz bei noch verdünnteren Lösungen (siehe Taf. VII) der entsprechend ebenfalls geringeren Azidität der van't Hoff' schen Lösung zufolge hier. in gleicher Weise die Gestalt der Kurve unbeeinflusst lässt. Gemäss dieser Anschauung würde dann die zunehmende Azidität der van't $\mathrm{H}$ off' schen Lösung mit ibrer Konzentration eine ansteigende Wirkung haben, die, vorher durch die intensiven Wirkungen der Salze superponiert, ihr Maximum der Wirksamkeit ungefähr bei oder kurz vor der Konzentration $B$ erreichen, später durch andere Salzwirkungen aber wieder zurückgedrängt werden wird. Die Richtigkeit dieser Anschauung ist indessen durch weitere Versuche noch zu erweisen.

Was nun die The orie dieser Giftwirkungen anbetrifft, so halte ich die Anzahl der hier gesehilderten Versuche noch bei weitem nicht für ausreichend, um über sie eine bestimmte Aussage machen zu können. Wie in der Einleitung erwähnt wurde, galt es zunächst, zu untersuchen, $o b$ die von den meisten Autoren angenommene, von Paul Bert ${ }^{1}$ ) besonders kräftig verteidigte Theorie von der rein "physikalischen" oder osmotischen Natur dieser Giftwirkungen tatsächlich der Wirklichkeit entspricht. Es ergibt sich nun deutlich aus den geschilderten Versuchen, dass dies auf keinen Fall zunächst in dem einfachen, von den betreffenden Autoren geglaubten Sinne

1) Siehe v. Fürth, Chemische Physiologie usw. S. 629. Jena 1903. 
der Wasserentziehung richtig ist. Vielmehr setzt sich die Wirkung, mehr entsprechend dem, was die „älteren Physiologen" glaubten, aus spezifischen, aber durchaus nicht in osmotischem Sinne zusammenwirkenden Einzeleffekten mit sehr charakteristischen und konstanten Unterschieden für die einzelnen Salze zusammen. Es ist nun auf der anderen Seite selbstverständlich und soll natürlich auch hier nicht unbeachtet gelassen werden, dass $\mathrm{n} e \mathrm{ben}$ diesen chemischen oder, besser gesagt, physikalisch-chemischen Wirkungen auch definitionsgemässe osmotische Vorgänge eine Rolle spielen. Die vorangehenden Ausführungen sollen sich nur gegen die meines Erachtens sehr unberechtigte, weil nur auf relativ wenige und nicht sehr tief eindringende Versuche sich gründende Verallgemeinerung von der "einfachen" osmotischen Natur der in Frage kommenden Erscheinungen wenden.

Auf zweierlei Tatsachen möchte ich indessen noch im Zusammenhang mit der Besprechung der osmotischen Theorie dieser Giftwirkungen aufmerksam machen.

Nehmen wir einstweilen die Angemessenheit der rein osmotischen Theorie, der nur wasserentziehenden Wirkungen konzentrierter Lösungen an, so ist zunächst folgende Einschränkung zu machen. Die Giftigkeit einer hypertonischen Lösung ist nicht direkt proportional ihrer Konzentration. Wenn ich auch das Gegenteil dieses Satzes in der entsprechenden bestimmten Form in der Literatur nicht habe finden können, so glaube ich doch keinen Fehler zu begehen, wenn ich sage, dass die Mehrzahl der Autoren (wie vor der Anstellung dieser Versuche ich selbst) dieser Ansicht war. Wäre diese Meinung aber richtig, so müsste die Kurve zunächst einer einzigen Salzlösung eine stetige, wenn auch vielleicht nicht geradlinige Form haben. Sämtliche hier gegebenen Kurven zeigen aber im Gegenteil einen ausgesprochenen, beim Seewasser z. B. ganz rapiden steilen Abfall bei einer gewissen Konzentration, der einem bestimmten kritisehen Werte der stetigen Wasserentziehung entspricht. Es gibt mit anderen Worten eine kritische Konzentration der Aussenlösung, bei welcher die vorber kaum nachweisbaren Gjftwirkungen hypertonischer Salzlösungen einen ganz ausserordentlich schnellen $\mathrm{Zuwachs}$ erreichen.

Nun ist indessen zweitens wichtig und in Betracht zu ziehen, dass eine osmotische Theorie dieser Giftwirkungen auch mit dieser 
Einschränkung den Tatsachen nicht vollständig entspricht. Denn es zeigt sich, dass dieser plötzliche Abfall der Kurven durchaus nicht bei gleicher osmotischer Konzentration der Aussenlösungen eintritt, sondern dass, wie der Vergleich der reinen $\mathrm{NaCl}$-Kurve mit der des Seewassers zeigt (Fig. 2), unter Umständen im Gegenteil die verdünntere ${ }^{1}$ ) Lösung bedeutend giftiger ist, d. h. den kritischen Punkt entsprechend ehererreicht. Auch selbst in diesem Fall handelt es sich also keineswegs um rein osmotische Vorgänge.

Für eine zureichende Erklärung dieser spezifischen Dynamik der Salze oder, was diesem entspricht, der charakteristischen Formen der Kurven halte ich nun die vorliegenden Versuche für nicht zureichend. Ich habe zwar, um speziell den Anteil rein osmotischer Vorgänge an diesen Giftwirkungen näher zu studieren, eine ziemliche Reihe von Versuchen mit Nichtelektrolyten, z. B. Rohrzuclier, angestellt, doch haben auch diese mir bis jetzt kein klares Bild der Verhältnisse geben können. Doch seien der Vollstäudigkeit halber die Versuche mit Rohrzucker hier angeführt. Die Kurve auf Taf. VII gibt die Durchschnittsresultate; die genauen Zahlen sind in Tabelle 20 (S. 594) enthalten.

In Worten ausgedrückt, zeigt es sich, dass

1. die Rohrzuckerkurve im allgemeinen eine beträchtliche Ähnlichkeit mit $\mathrm{NaCl}$ besitzt, dass aber

2. $\mathrm{NaCl}$, namentlich in isotonischer Konzentration mit Rohrzucker, deutlich giftiger wirkt als Rohrzucker.

Dies letztere kann aus Taf. VII ersehen werden. Nur ist darauf aufmerksam zu machen, dass die zwei Kurven nicht in isotonischer Lage eingetragen worden sind. Um das letztere zu erreichen, muss man die Rohrzuckerkurve sich ungefähr um $1 \frac{1}{2}$ Felder nach rechts verschoben denken, so dass z. B. Punkt $S$ der Rohrzuckerkurve Punkt $C$ der $\mathrm{NaCl}-K u r v e$ entspricht. (Am rechten Ende muss noch $\mathrm{mehr}$ als $1^{1 / 2}$ Feld verschoben werden.) Es zeigt sich dann beim

1) Diese Tatsache würde noch bei weitem auffälliger werden, wenn man die Giftigkrit isosmotischer NaCl- und Seewasserlösungen (also nicht entsprechend Fig. 2) gemeinsam in ein Feld eintragen würde. Da nämlich $\mathrm{NaCl}$ von allen in Betracht kommenden Salzen das kleinste Molekulargewicht hat, so würde dadurch die Disproportionalität zwischen osmotischer Konzentration und Giftigkeit resp. die intensive Giftigkeit des reinen $\mathrm{NaCl}$ noch deutlicher als in Fig. 2 zutage treten. 
Vergleich der übereinanderstehenden Zahlen ausserordentlich auffällig die geringere Giftigkeit des Rohrzuckers. Als Beispiele solcher Unterschiede seien folgende Zahlen angeführt:

Rohrzucker 105 Mil. 96 Min. 78 Min. - - 51 Min. $\mathrm{NaCl}$
$\mathrm{N}$

Tabelle 20. Rohrzucker.

\begin{tabular}{|c|c|c|c|c|}
\hline \multicolumn{2}{|r|}{ Konzentration } & $\begin{array}{l}\text { Lebenszeit des } \\
\text { Weibchens } \\
\text { in Minuten }\end{array}$ & $\begin{array}{l}\text { Lebenszeit des } \\
\text { Männchens } \\
\text { in Minuten }\end{array}$ & $\begin{array}{c}\text { Mittlere } \\
\text { Lebenszeit }\end{array}$ \\
\hline & $\frac{9}{8}$ 1) $n$. & 37 & 48 & 42,5 \\
\hline $90 \mathrm{c}$ & $\left.\mathrm{cm} \frac{9}{8}+10 \mathrm{ccm} \frac{5}{8} 2\right)$ & 38 & 53 & 45,5 \\
\hline 80 & $" \frac{9}{8}+20 \quad " \frac{5}{8}$ & 43 & 58 & 50,5 \\
\hline 70 & $" \frac{9}{8}+30 \quad " \frac{5}{8}$ & 48 & 62 & 55,0 \\
\hline 50 & $" \frac{9}{8}+50 \Rightarrow \frac{5}{8}$ & 59 & 78 & 66,5 \\
\hline 30 & $" \frac{9}{8}+70 " \frac{5}{8}$ & 69 & 87 & 78,0 \\
\hline 10 & $\Rightarrow \frac{9}{8}+90 \quad, \frac{5}{8}$ & 78 & 114 & 96,0 \\
\hline & $\frac{5}{8} \mathrm{n}$ & 81 & 128 & 104,5 \\
\hline
\end{tabular}

Es ist möglich (und deshalb soll auf das zweite Resultat kein allzu grosser Wert gelegt werden), dass eine $5 / 8 \mathrm{n}$. und eine $9 / 8 \mathrm{n}$. Rohrzuckerlösung, obschon von J. Loeb in ähnlichen Verhältnissen oft angewendet, nicht vollkommen genau isotonisch mit Seewasser von $23,6 \%$ resp. $47,2 \%$ ist. Die rechnerische Überlegung ergibt allerdings eine annähernde Übereinstimmung, doch ist die Berücksichtigung des Dissoziationsfaktors im zweiten Falle nur schätzungsweise geschehen. Immerhin scheint aber die besprochene Abweichung grösser zu sein, als nach den Feblergrenzen zu erwarten ist. Genauere Versuche baben diesen Punkt zu entscheiden.

Am wahrscheinlichsten kommt es mir vor, dass die deletäre Wirkung solcher hypertonischer Lösungen in einer Koagulation

1) Annähernd isotonisch mit doppeltkonzentr. Seewasser:

2) Annähernd isotonisch mit Oaklandseewasser. 
des Plasmas zu suchen sein wird. Und zwar kann diese Ausfällung hervorgerufen werden

entwe der durch eine bestimmte, durch die osmotische Wasserentziehung bedingte Konzentrationserhöhung der im Innern del Zellen gelösten Elektrolyte

oder durch Eindringen von Salzen durch die Membranen,

oder aber - was am besten mit den beobachteten Tatsachen übereinstimmen würde - durch gleich zeitige Wirkungen beiderlei Art.

Für diese Ansicht spricht zunächst der bei allen Kurven zu beobachtende mehr oder weniger steile Abfall. Ferner sind in der Chemie der kolloidalen Lösungen sehr wohl spezifische Wirkungen einzelner Salze, welche unter Umständen, namentlich je nach der Reaktion des Eiweisses, auch antagonistisch sich verhalten können ${ }^{1}$ ), bekannt. Allerdings muss hier darauf hingewiesen werden, dass in der physikalischen Chemie der Kolloide selbst diese Gesetzmässigkeiten noch nicht in einem Masse aufgeklärt worden sind, dass eine Anwendung derselben für biologische Zwecke zu sicheren Ergebnissen führen würde. Endlich sprechen für die Koagulationsnatur dieser Lösungswirkungen auch die für den umgekehrten Fall angestellten, oben öfters zitierten Versuche von J. Loeb, bei welchen ein ähnlicher kritischer Punkt der verminderten Aussenkonzentration und ebenfalls darauf ein steiler Abfall beobachtet wurden, - ein Verhalten, das der Koagulation von Solen, welche nur beim Vorhandensein gewisser Salze als solche bestehen können, entsprechen würde.

Ich hoffe, an einem günstigeren Objekte, z. B. an Medusen, durch Bestimmung des Wasserverlustes bei versehiedenen Konzentrationen usw. Beiträge zur Lösung dieser Frage geben zu können.

Anhangsweise sei hier einiger Versuche gedacht, welche noch eine andere Wirkung verschieden konzentrierter Lösungen auf meine Versuchstiere ergaben. Bei den Versuchen über die verbessemde Wirkung geringer Mengen $\mathrm{Na}_{2} \mathrm{CO}_{3}$ stellte es sich heraus, dass die mit einigen Tropfen Phenolphthalein gefärbten Lösungen von gleichem Volum und gleicher Alkalinität sich verschieden schnell entfärbten. Wurde z. B. $\frac{6}{8}$ n. NaCl-Lösung durch wenige cem $\mathrm{Na}_{2} \mathrm{CO}_{3}$ alkaliseh

1) Siehe z. B. Höher, Physik. Chemie der Zelle S. 164 ff. Leipzig 1902. 
gemacht, ein Teil der Lösung verschieden stark verdünnt, gleiche Volumina genommen und gleich viel Tiere (etwa 20 in ein Reagenzglas) hineingesetzt, so konnte schon nach einer Viertelstunde ein Unterschied in der Färbung wahrgenommen werden. Regelmässig entfärbte sich die konzentriertere Lösung zuerst. Nach zirka einer halben Stunde, nach welcher Zeit bei dieser Versuchsanordnung die Tiere in allen Versuchsgläsern noch am Leben sind, ist die konzentrierteste Lösung oft vollständig entfärbt. Es wird also mit anderen Worten in den konzentrierteren Lösungen mehr einer Säure ausgeschieden, deren chemische Natur (ob, was am wahrscheinlichsten ist Kohlensäure, oder aber auch Milchsäure, Harnsäure usw.) noch nicht festgestellt werden konnte. Bei längerem Stehen kann sich natürlich der Unterschied zwischen den einzelnen Konzentrationen, namentlich infolge des früheren Todes in den höheren Konzentrationen wieder verwischen, ein Verhalten, das indessen nie vollständig beobachtet wurde.

Diese Versuche sollen erweitert und fortgesetzt werden.

\section{Zusammenfassuug.}

Die Hauptresultate dieser Untersuchung sind folgende:"

1. Es wurde eine möglichst genaue Kurve der Giftigkeit von Seewasser für Süsswasser-Gammarus bis zur Konzentration 51,9\%00 gemessen, welche sich insbesondere als verschieden für beide Geschlechter erwies (die Männchen resistenzfähiger als die Weibchen).

2. Es wurde diese Giftwirkung des Seewassers an der Hand entsprechender Kurven in bezug auf den Anteil der einzelnen Salze untersucht. Und zwar wurde von Einzellösungen ausgegangen und durch systematische Untersuchung der möglichen und in Betracht kommenden Variationen und Kombinationen die Wirkung des Seewassers analysirt resp. durch allmähliche Vereinigung sämtlicher wichtiger im Seewasser vorhandener Salze zur van't Hoff'schen Lösung aufgebaut. Die Wirkung der einzelnen Salze ergab sich dabei folgendermassen:

$\mathrm{NaCl}$ in der entsprechenden Konzentration wie im Seewasser vorbanden ist bei weitem giftiger als das relativ konzentriertere Seewasser.

$\mathrm{KCl}$ in Kombination mit NaCl verringert die Giftigkeit desselben, und zwar am stärksten von allen anderen Salzen. Dies zeigt 
sich zunächst in der Vergrösserung der Lebenszeiten sowie fernerhin im Hinausschieben des ausführlich beschriebenen charakteristischen Abfalles der Kurven.

$\mathrm{CaCl}_{2}$ hat einen ähnlichen, wenn auch nicht so starken Effekt wie $\mathrm{KCl}$.

$\mathrm{MgS} \mathrm{O}_{4}$ verbessert ebenfalls die Giftigkeit des $\mathrm{NaCl}$, wenn auch in noch schwächerem Masse als $\mathrm{CaCl}_{2}$. Ausserdem hat $\mathrm{MgSO}_{4}$ einen charakteristischen und: spezifischen Einfluss auf die Gestalt der Kurven insofern, als es den Abfall derselben in all e n Lösungen, in welchen es vorhanden ist, schwächt resp. verlangsamt.

Diese drei Salze wirken also deutlich antagonistisch in bezug auf die Giftigkeit des $\mathrm{NaCl}$. Im Gegensatz hierzu verstärkt

$\mathrm{MgCl}_{2}$ sowohl in Kombination mit $\mathrm{NaCl}$ allein als auch in jeder anderen Zusammenstellung ${ }^{1}$ ) deutlich die Giftigkeit des NaCl.

Die Reihenfolge der optimalen Lösungen ist in synthetischer Reihenfolge diese:

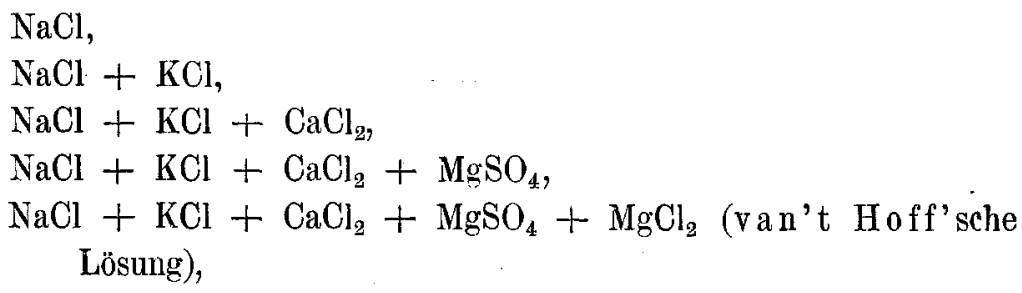

Seewasser ${ }^{2}$ ).

Einige kleinere Abweichungen der Giftigkeit der van't $\mathrm{H}$ off schen Lösung von der des Seewassers sind teils auf Versuchsfehler, teils aber, wie durch entsprechende Versuche gezeigt werden konnte, auf die schwache Azidität der van't Hoff'schen Lösung im Gegensatz zu der merklich alkalischen Reaktion des verwendeten Seewassers zurückzuführen.

3. Für die mögliche Theorie dieser Giftwirkungen muss auf den ausführlichen Text verwiesen werden. Die Versuche ergeben im allgemeinen aber deutlich, dass die Giftwirkungen des Seewassers auf Süsswassertiere keineswegs, wie bisher geglaubt und speziell von Paul Bert behauptet worden ist, rein „physikalischer", d. h. osmotischer Natur sind, sondern dass es sich hier, analog wie in dem

1) Mit Ausnahme der van't Hoff'schen Lösung, d. h. einer Kombination aller Seesalze.

2) Siehe Tafel. 
598 Wolfgang Ostwald: Versuche über die Giftigkeit des Seewassers etc.

von J. Loeb untersuchten Falle der Wirkungen von Süsswasser auf Seetiere, um spezifisch chemische respektive physikalisch-chemische, selbstverständlich mit osmotischen Vorgängen verknüpfte Prozesse handelt. Aller Wahrscheinlichkeit nach bestehen diese schädlichen Prozesse in Koagulationswirkungen von Salzen.

Anhangsweise wird über ein paar vorläufige Versuche berichtet, welche ergaben, dass eine $\mathrm{Säureabscheidung}$ von seiten der Krebse, deren chemische Natur aber $\left(\mathrm{ob} \mathrm{CO}_{2}\right.$ oder Milchsäure, Harnsäure usw.) noch nicht festgestellt worden ist, stark zunimmt mit steigender Konzentration der Lösungen und so vielleicht auch einen Anteil an den toxischen Effekten der Salze hat. -

Zum Schlusse freue ich mich, auch an dieser Stelle Herrn Prof. J. Loeb für vielfachen Rat und freundliches Interesse während dieser Untersuchungen danken zu können. 
Archiv für die ges. Physiologie. Bd. 106.

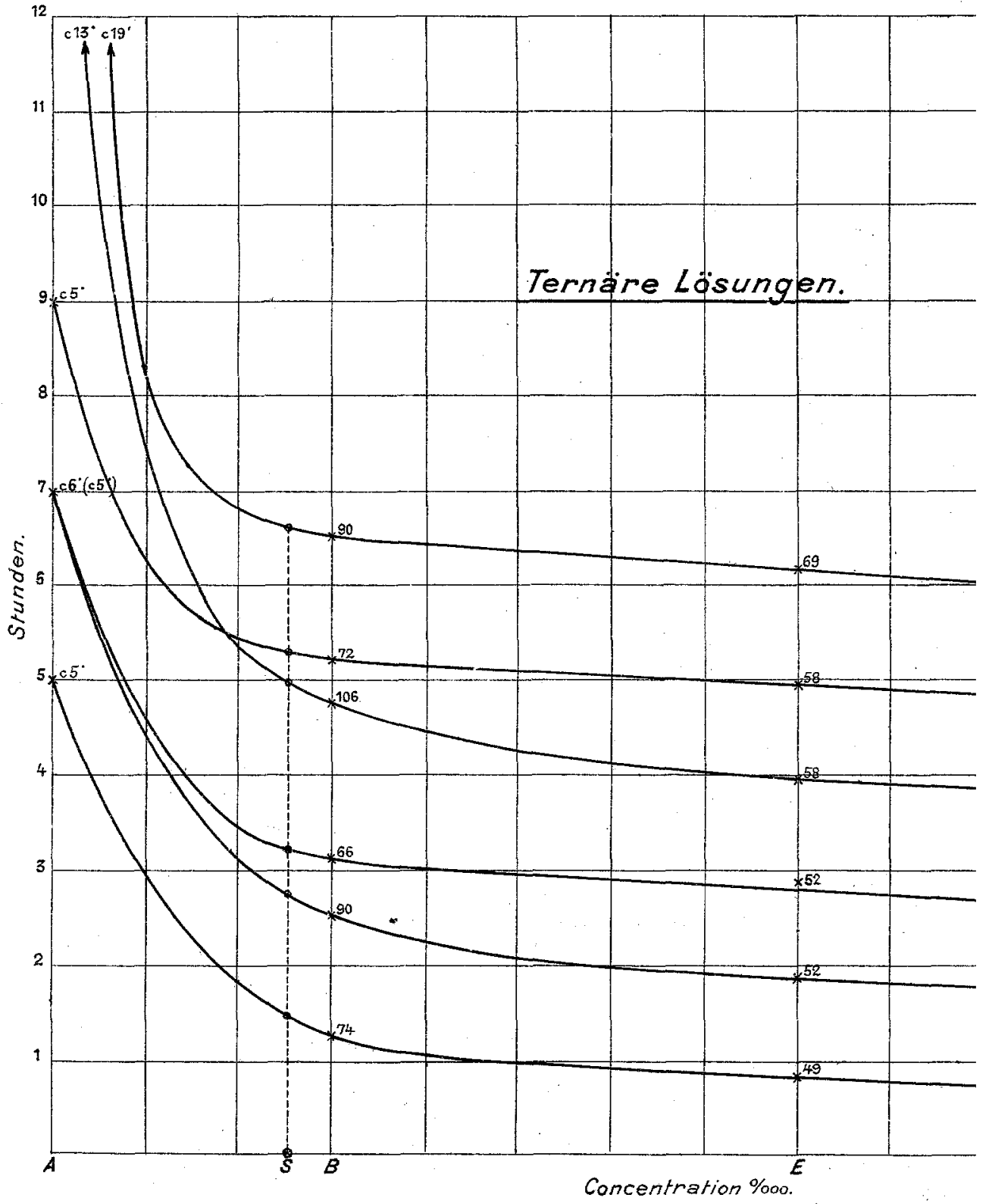


Archiv für die ges. Physiologie. Bd.106.

Taf. v

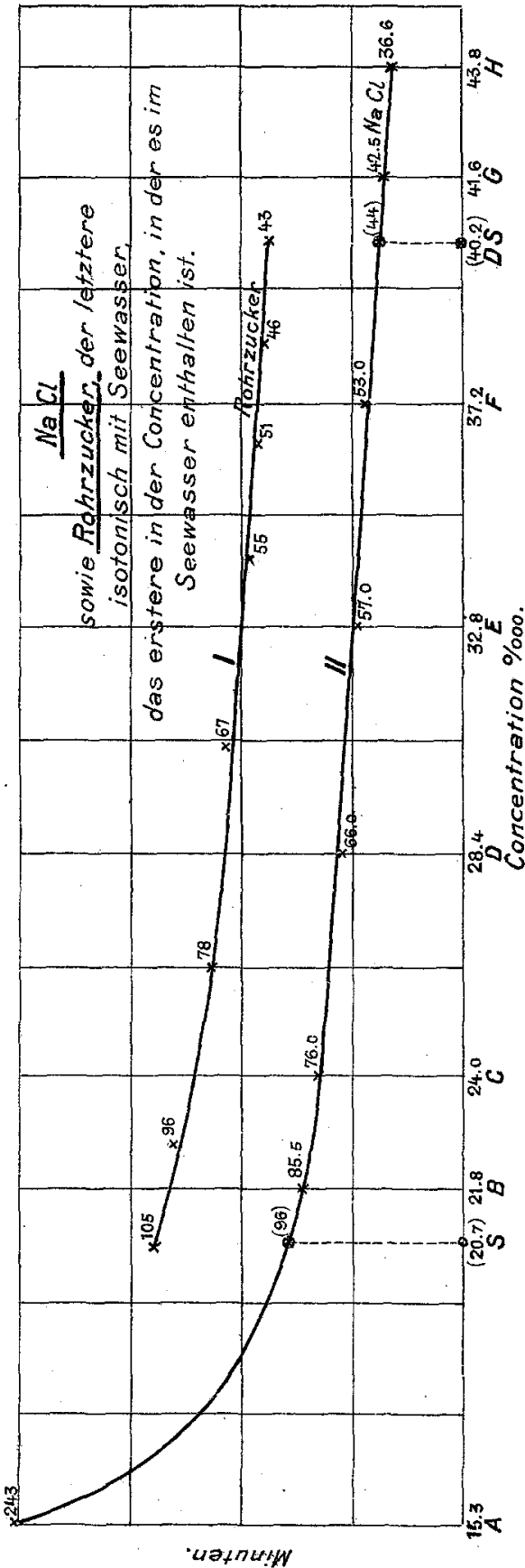

lith Anst.v.F.Wirtz,Darmstadt.

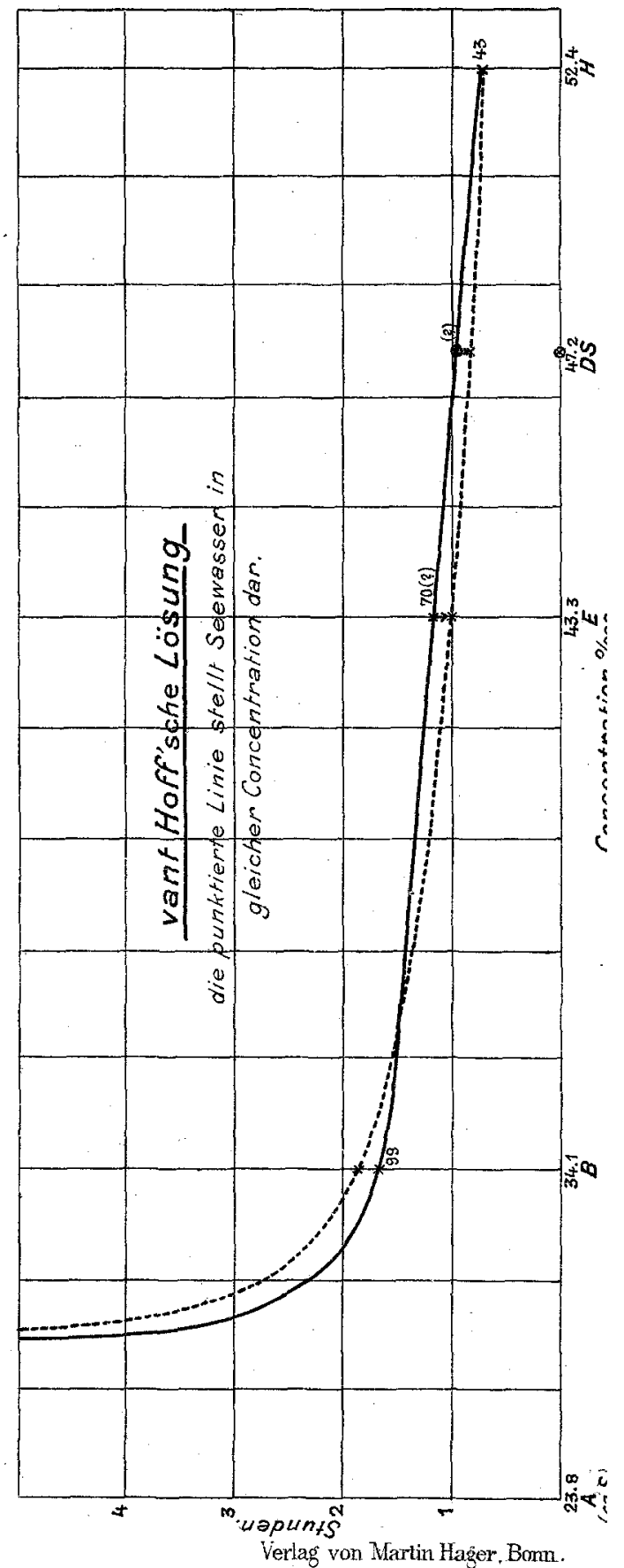


Archiv für die ges. Physiologie. Bd.106.

Taf. II.

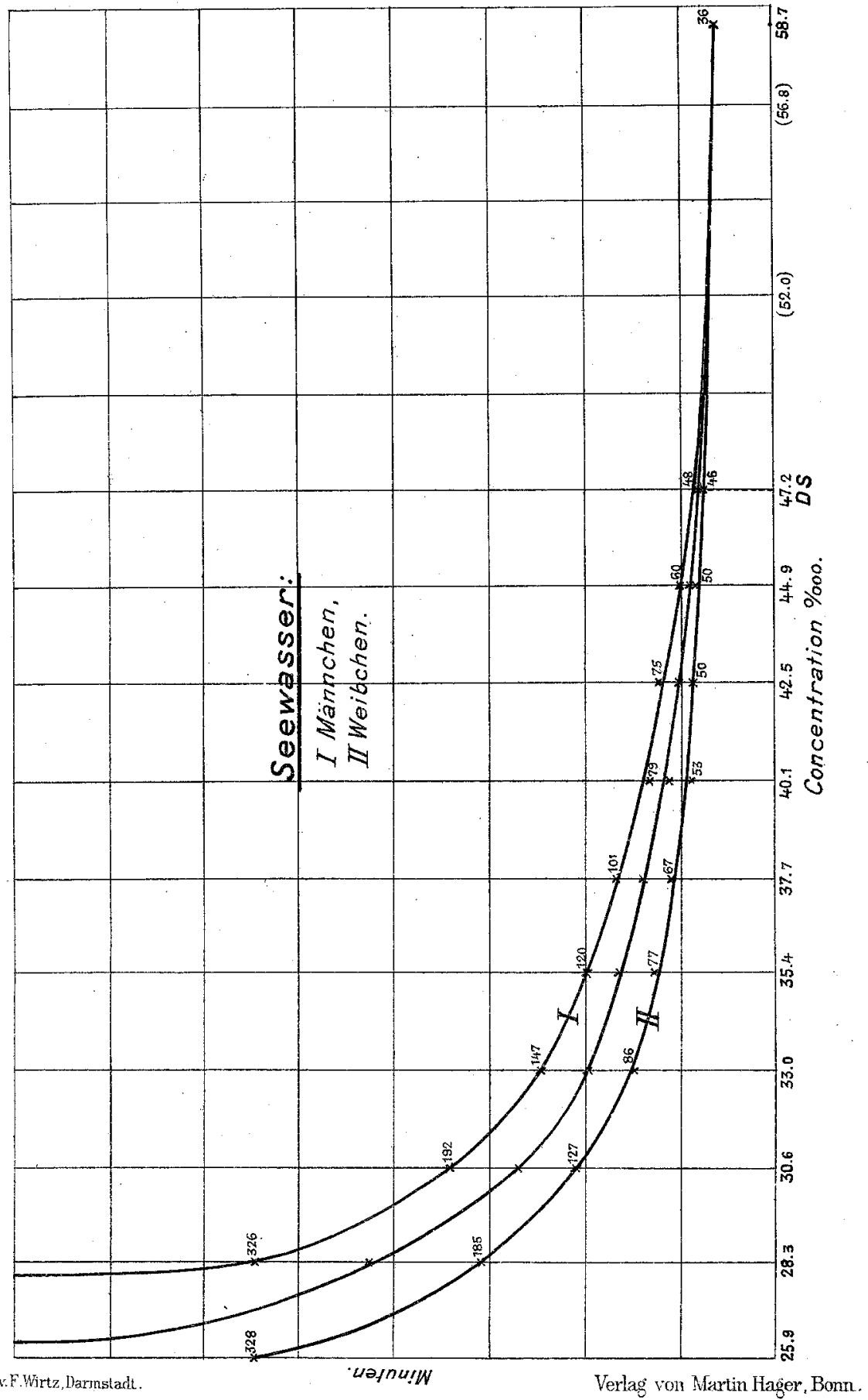


Archiv für die ges. Physiologie. Bd. 106.

Taf. III.

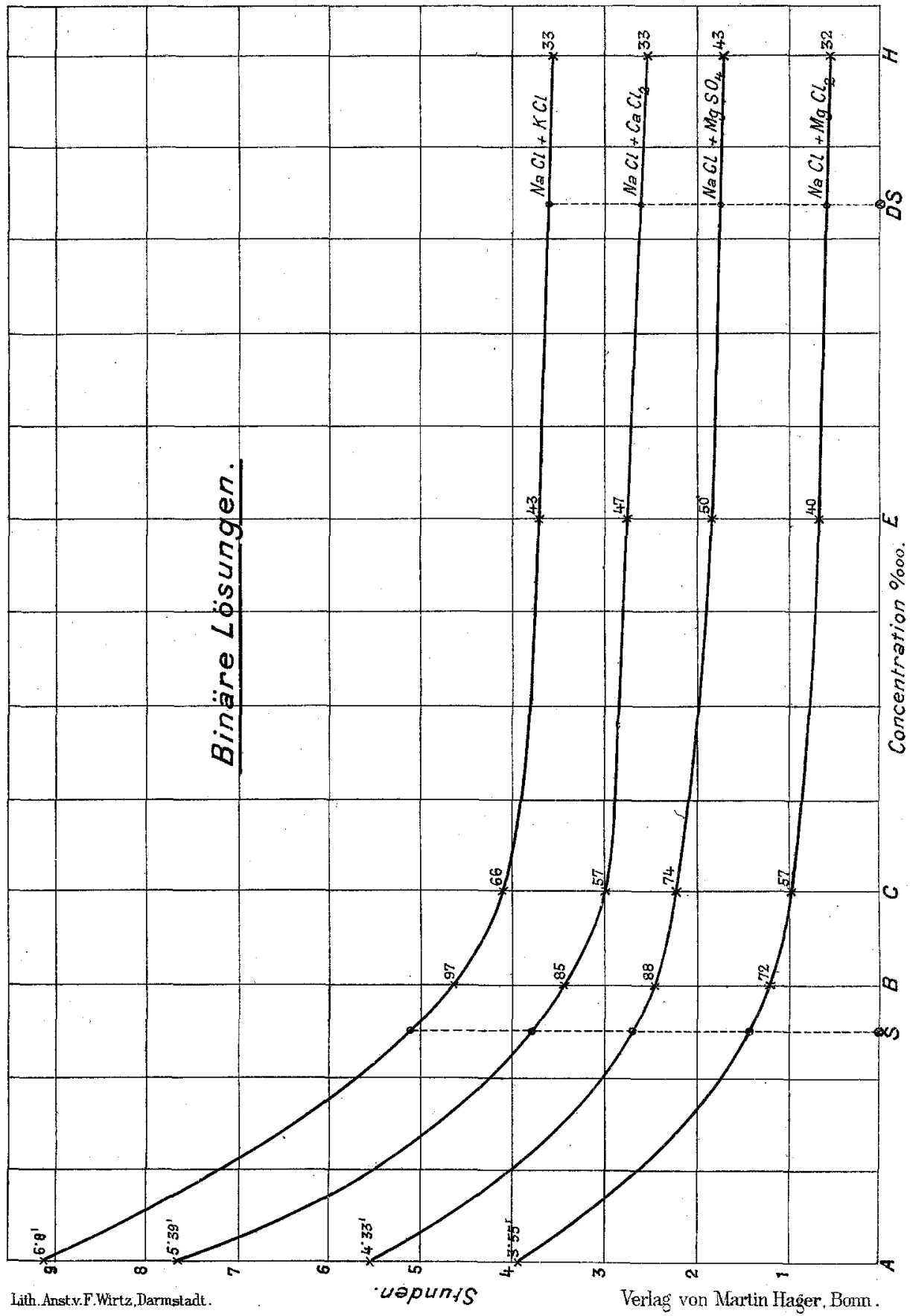


Archiv für die ges. Physiologie. Bd.106.

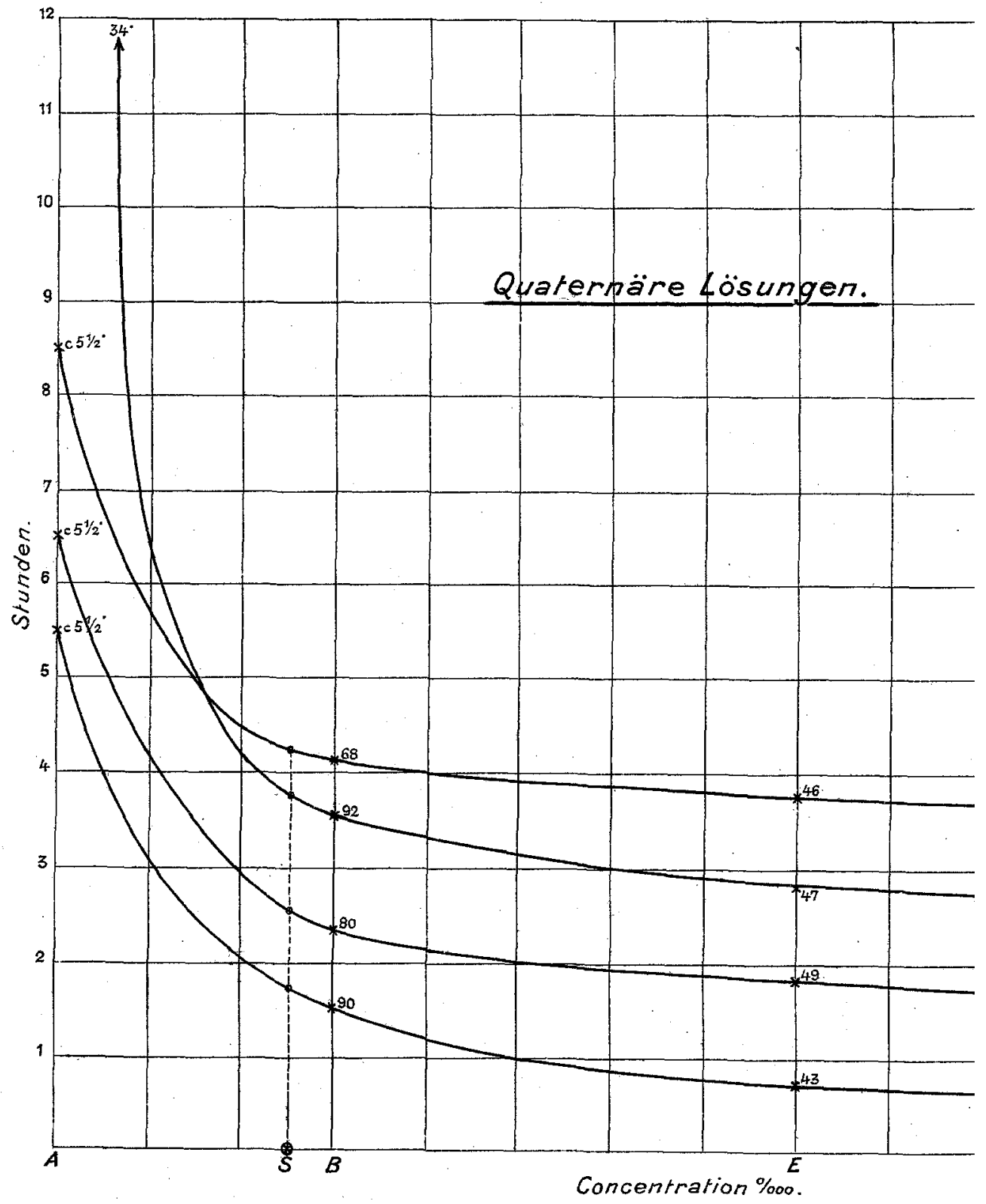

- Lith.Anstw.F.Wirtz, Darmstadt 


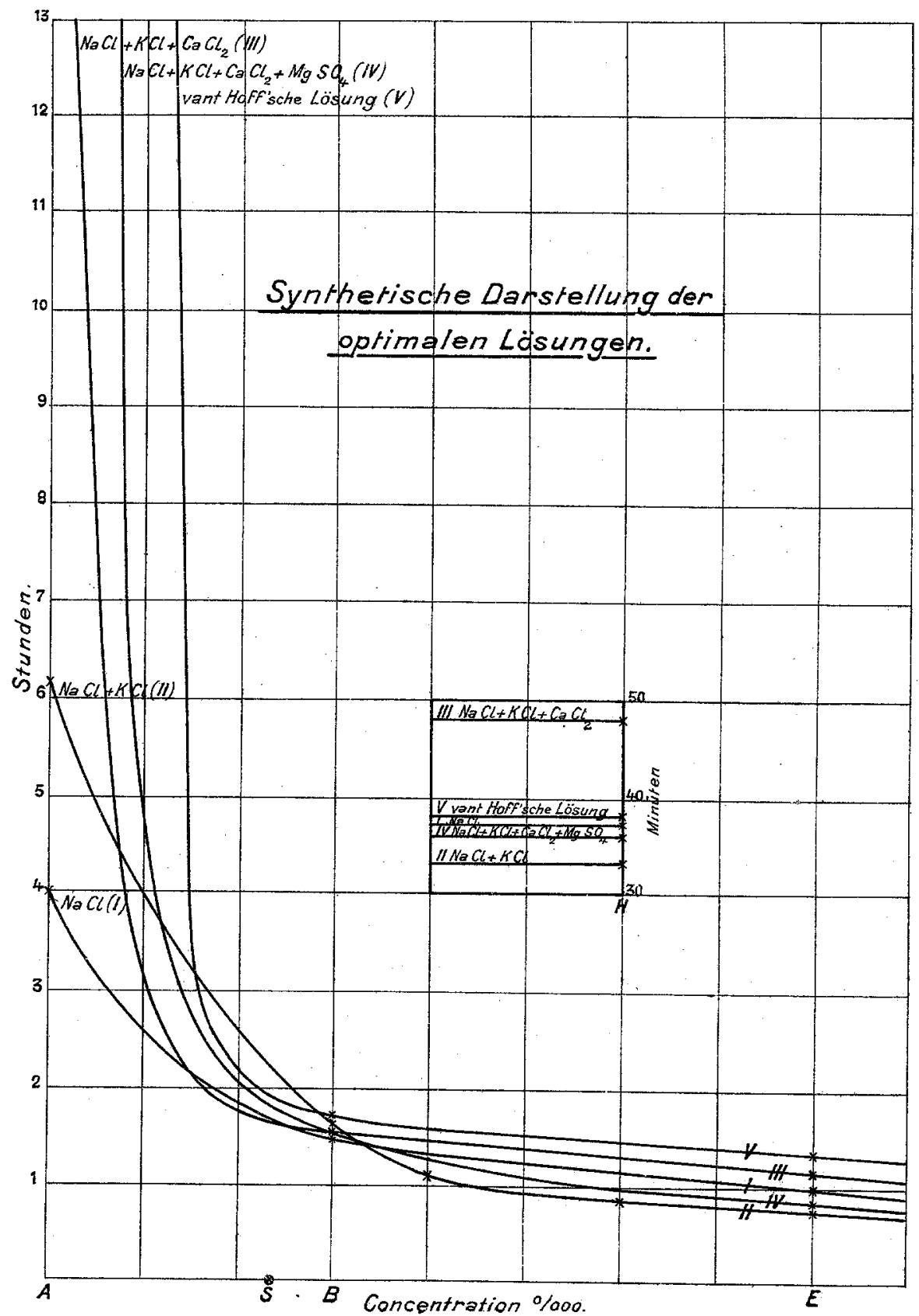

